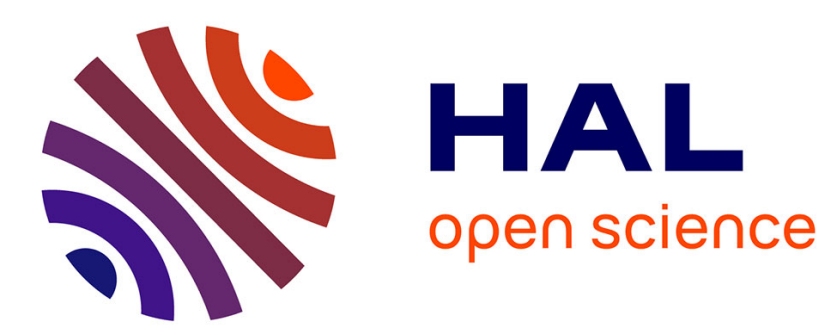

\title{
Les archives de la composante d'archéologie orientale de l'UMR 7044
}

\author{
Marie Stahl, Dominique Beyer
}

\section{To cite this version:}

Marie Stahl, Dominique Beyer. Les archives de la composante d'archéologie orientale de l'UMR 7044.

Archimède: archéologie et histoire ancienne, 2015, 2, pp.20-40. halshs-01587173

\section{HAL Id: halshs-01587173 \\ https://shs.hal.science/halshs-01587173}

Submitted on 13 Sep 2017

HAL is a multi-disciplinary open access archive for the deposit and dissemination of scientific research documents, whether they are published or not. The documents may come from teaching and research institutions in France or abroad, or from public or private research centers.
L'archive ouverte pluridisciplinaire HAL, est destinée au dépôt et à la diffusion de documents scientifiques de niveau recherche, publiés ou non, émanant des établissements d'enseignement et de recherche français ou étrangers, des laboratoires publics ou privés. 


\section{ARCHIMĖDE N $N^{\circ} 2$}

\section{DOSSIER THÉMATIQUE : ARCHIVES DE L'ARCHÉOLOGIE}

1 Marie STAHL

Introduction : la mémoire retrouvée des archéologues

9 Marie STAHL, Lucile SCHIRR

Les archives de l'archéologie : définition, législation, état des lieux

20 Dominique BEYER, Marie STAHL (collab. Catherine DUVETTE, Isabelle WEYGAND, Françoise LAROCHE-TRAUNECKER, Marie-José MORANT, Philippe QUENET) Les archives de la composante d'archéologie orientale de I'UMR 7044

41 Cassandre HARTENSTEIN

Le fonds Montet et la statue «maussade » de Ramsès II au Palais universitaire de Strasbourg

51 Soline MORINIÈRE

Les archives de I'archéologie au SRA Alsace : état des lieux et des fonds

59 Anne ROHFRITSCH

Les archives dites « manuscrites » des membres de l'École française d'Athènes :

l'exemple des « Strasbourgeois 》 (1846-1960)

66 Cécile COURTAUD, Isabelle LESUEUR, Soline MORINIÈRE, Juliette RÉMY, Bernadette SCHNITZLER, Marie STAHL, Georges TRIANTAFILLIDIS

Un projet collectif de recherche autour du fonds Arthur Stieber

78 Soline MORINIÈRE

La gypsothèque de I'Université de Strasbourg : quand les statues parlent d'elles-mêmes

\section{LA CHRONIQUE D'ARCHIMÈDE}

94 Frédéric COLIN (éd.)

La Chronique d'Archimède. Bilan des activités scientifiques 2014-2015 de I'unité mixte de recherche 7044

\section{VARIA}

134 Sarah DERMECH

Couleurs, éclat et brillance des crânes surmodelés : le cas du Néolithique Proche-oriental

150 Cinzia BEARZOT

La violence de l'État. La condamnation à mort sans jugement dans la Grèce ancienne

160 Doris MEYER

Jusqu'au dernier mot. Martyr, débat public et résistance dans la littérature de I'Antiquité tardive et à Byzance

170 Vincent PUECH

Les biens fonciers des élites sénatoriales à Constantinople et dans ses environs (451-641)

194 Clara MILLOT

Entre les enfants d'Hérodote et les enfants d'Adam Smith. Pour une approche économique des données archéologiques

Retrouvez tous les articles de la revue ARCHIMĖDE sur :

http://archimede.unistra.fr/revue-archimede/archimede-2-2015 


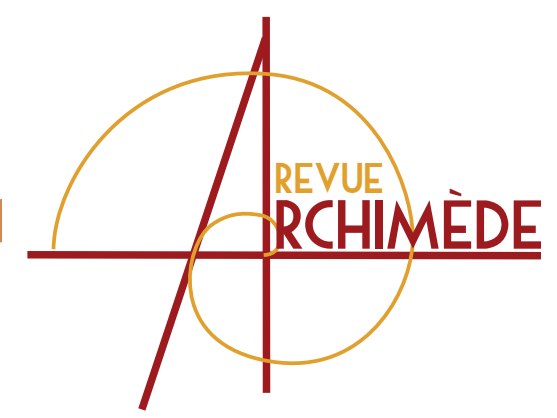

\section{LES ARCHIVES DE LA COMPOSANTE D'ARCHÉOLOGIE ORIENTALE DE L'UMR 7044}

\author{
Marie STAHL \\ Chargée d'études documentaires \\ DRAC Alsace, UMR 7044 Archimède \\ marie.stahl@culture.gouv.fr

\section{Dominique BEYER} \\ UMR 7044 Archimède \\ dominique.beyer@misha.fr
}

avec la collaboration de

Catherine DUVETTE

CNRS, UMR 7044 Archimède

Françoise LAROCHE-TRAUNECKER

Chercheur associé, UMR 7044 Archimède

Marie-José MORANT

Ingénieur-documentaliste retraitée

UMR 7044 Archimède

Philippe QUENET

Professeur, Université de Strasbourg CNRS-UMR 7044 Archimède

Isabelle WEYGAND

RÉSUMÉ

Chercheur associé, UMR 7044 Archimède

La documentation scientifique des missions orientales de I'UMR 7044 bénéficie d'un local commun dédié à l'archivage au sein de la MISHA. Un inventaire sommaire des fonds liés à ces opérations archéologiques a été entrepris afin de fournir aux chercheurs une vision d'ensemble de ces collections.

Huit missions ont ainsi été répertoriées. Pour la Turquie : Porsuk, Gülnar et la mission relative aux villes de Lycie ; pour la Syrie : Tell Hariri / Mari, Ramadi, Tell Mashnaqa, Meskéné / Emar et la mission archéologique syro-française de la Syrie du Nord. À cela s'ajoute un fonds de chercheur: celui de Jacqueline Pirenne, de même que des séries à but pédagogique et les collections d'étude : plaques de verre et diapositives de cours, empreintes de sceaux et de scellements de porte et moulages.

Chaque lot figure un cas particulier de création et de détention d'archives, et témoigne de la multiplicité des supports et formats uti-

\section{MotS-CLÉS}

Archéologie orientale, archives scientifiques, archives de l'archéologie, documentation archéologique, archives de la recherche, gestion des archives, ministère de l'enseignement supérieur et de la recherche, conservation,

valorisation. lisés. Ces archives de la recherche en archéologie représentent des objets muséables et collections à valoriser, tant pour les données susceptibles d'être réutilisées que pour afficher une histoire de la discipline.
Scientific documentation of the UMR 7044 ancient Orient archaeological missions benefits from a space specifically dedicated to archiving inside of the MISHA building. A brief inventory of these archives has been undertaken in order to give the researchers a general overview of the collections.

Eight missions have been registered. Turkey: Porsuk, Gülnar, «Cities of Lycia» ; Syria: Tell Hariri / Mari, Ramadi, Tell Mashnaqa, Meskéné / Emar and the archaeological French-Syrian mission of Northern Syria. Research archives of Jacqueline Pirenne, as well as pedagogic collections can be added to the inventory: glass plates and slides, seal prints and mouldings.

Each series represents a particular case of archival management and shows the diversity of forms and mediums. These archaeological archives can be viewed as museum objects and as parts of collections to be enhanced, in order to revisit the datas or trace back the history of archaeology.
KeYWORdS Scientific records, archaeological documentation, oriental Archaeology, archaeological archives, archaeological records, research archives, records management, Ministry of education and research, preservation, development. 
L'UMR 7044 «Archéologie et histoire ancienne : Méditerranée - Europe » (Archimède) a incontestablement bénéficié d'une situation privilégiée lors de la phase d'établissement du programme de la Maison interuniversitaire des sciences de I'homme - Alsace (MISHA). Le responsable du projet, Alain Chauvot, étant membre de notre équipe, un certain nombre d'aménagements spécifiques ont pu être plus facilement obtenus, en particulier pour les activités des archéologues, avec par exemple des salles de traitement de matériel archéologique, des salles de conservation de collections, etc. Une grande salle a été alors prévue, dès le départ, pour servir essentiellement de salle d'archives pour les diverses missions qui n'avaient pas d'autres lieux de stockage par ailleurs. Les missions en Orient en font partie.

Le fonds de la composante orientale de I'UMR 7044 Archimède concerne une série d'opérations d'archéologie programmée, effectuées par les enseignants-chercheurs de I'université, auxquelles s'ajoutent diverses collections documentaires, dons, dépôts, agrégés au fil des changements d'affectation des membres des équipes, mais également grâce à des relations de travail ou familiales.
Si les grandes catégories, déjà détaillées dans l'article consacré aux archives de fouille d'opération d'archéologie préventive, se retrouvent ici produites à l'identique, le concept même de documentation scientifique s'étend plus largement à toute la gestion administrative des différentes campagnes, aux publications résultant des opérations de terrain, et aux multiples supports pédagogiques destinés aux cours magistraux et travaux dirigés des enseignants. Il paraît évident, faut-il le préciser ici, que cet article ne donnera au lecteur qu'une vision partielle de ce fonds, qui ne reflète pas toute sa richesse ni sa variété en terme de typologie documentaire. Il permettra toutefois, nous I'espérons, de fournir un premier état des lieux sous la forme d'un inventaire sommaire par grandes séries, identifiant les sites principaux et indiquant une volumétrie globale. Cet inventaire tentera donc essentiellement de montrer tout le potentiel que recèle ces collections, d'une part, en terme d'exploitation des contenus scientifiques (sources primaires non publiées, revisite des données, etc.) et, d'autre part, en vue de la valorisation ultérieure d'un patrimoine documentaire au sein d'un établissement d'enseignement supérieur et de recherche.

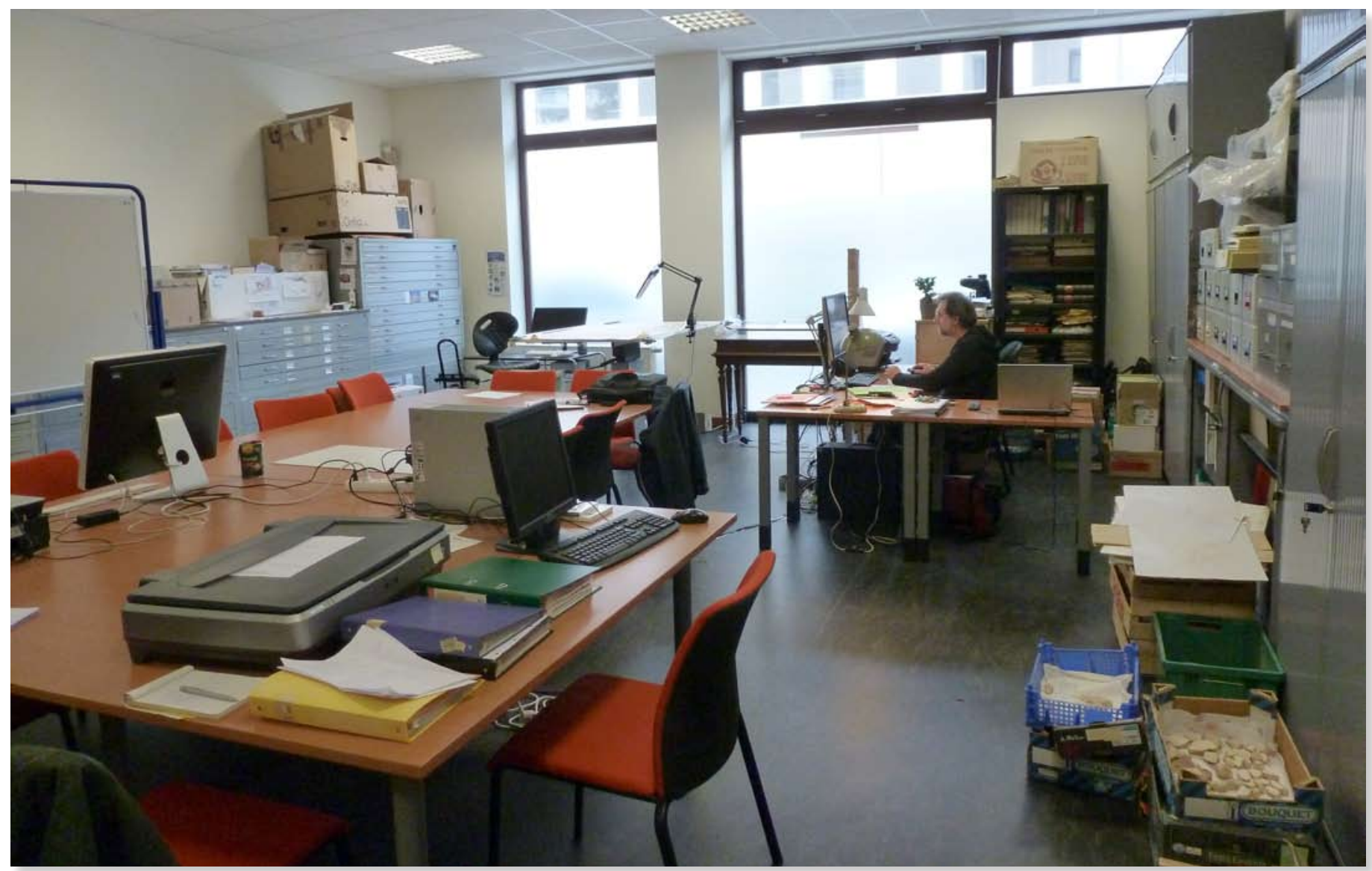




\section{1 - UN ÉTAT SOMMAIRE DES COLLECTIONS DE L'ORIENT ANCIEN À L'UMR ARCHIMĖDE}

\subsection{PORSUK (TURQUIE): UNE \\ TRANSMISSION DES ARCHIVES D'UN \\ DIRECTEUR À SON SUCCESSEUR}

Le site de Porsuk, en Cappadoce méridionale, a été fondé aux environs de 1600 av. J.-C. et occupé durant toute la période hittite. Fouillé par Olivier Pelon (Maison de I'Orient méditerranéen (MOM, Lyon) de 1969 à 2003, avec plusieurs interruptions, la direction du chantier est assurée depuis par Dominique Beyer (MISHA, Strasbourg). La publication de ce site est en cours à I'UMR dans le programme Territoires et empires d'Orient, axe Les sites et leur environnement, opération La Cappadoce méridionale du Bronze récent à la période romaine [1].

Lors du changement de direction et surtout après le décès d'Olivier Pelon en 2013, les archives scientifiques liées à Porsuk ont été récupérées progressivement et transférées de Lyon à la MISHA. Une partie non quantifiable resterait encore au domicile d'Olivier Pelon : sa veuve serait favorable à un versement des documents

Figure 2

Aperçu de la documentation scientifique des fouilles de Porsuk (cliché : Soline Morinière / DRAC Alsace)

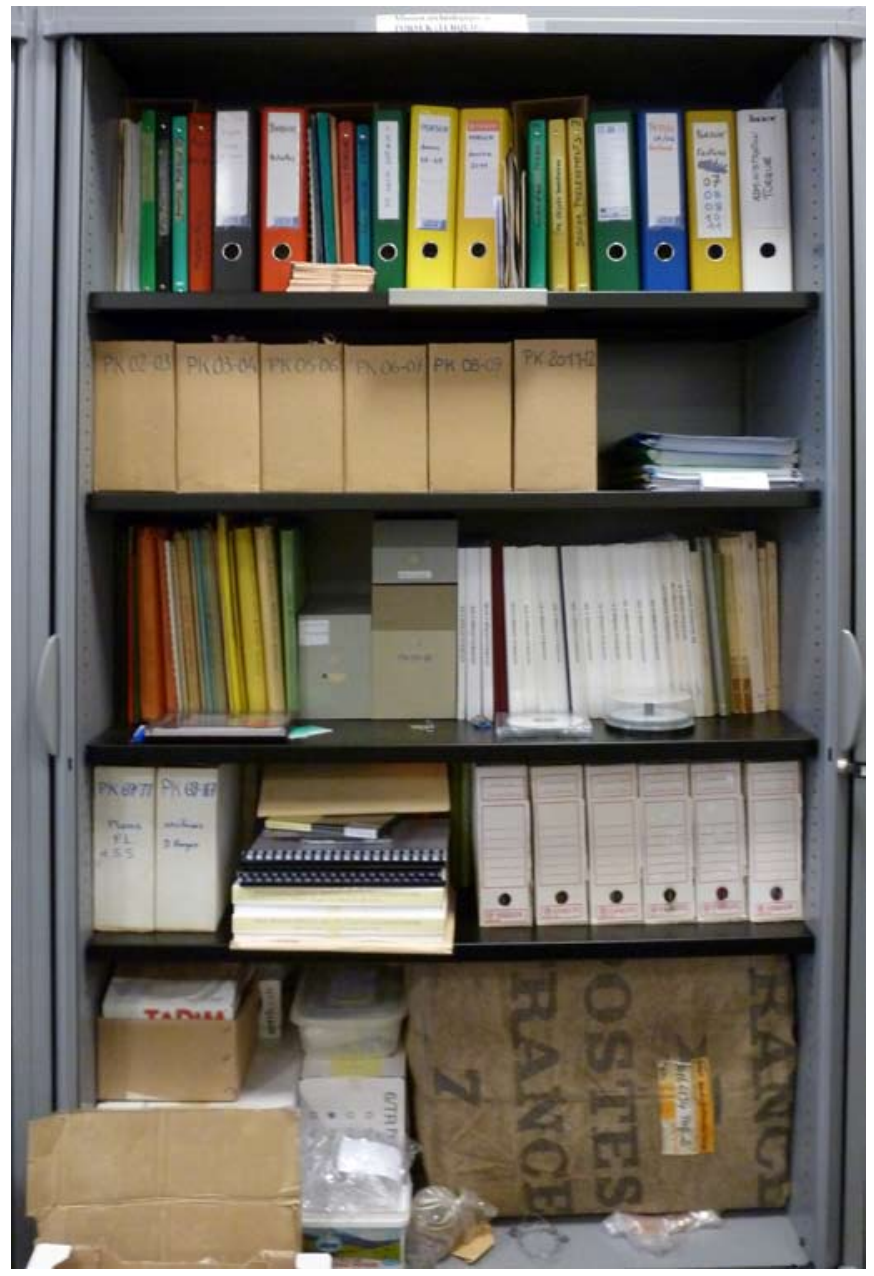

à la MISHA. La collection est stockée actuellement dans la salle dédiée aux archives scientifiques de I'UMR.

Deux séries correspondant aux directions successives se détachent nettement dans les armoires et étagères, mais de nombreux documents anciens ont été intégrés dans le classement des fouilles en cours, pour les besoins de la recherche. Un rangement par types de document a été effectué, même si, nous le verrons, la frontière s'avère moins nette lorsqu'on aborde les chantiers en cours de publication par des membres de l'équipe.

La série fait figure de cas d'école. Toutes les grandes catégories de documents sont représentées, montrant l'évolution des techniques de prises de vues et de notes de chantier depuis 1969 :

- documentation administrative : gestion de chantier, liste des équipes, correspondance, etc. 5 classeurs et 6 boîtes d'archives ;

- analyses et datation : 4 classeurs d'analyse et environ 1 mètre linéaire $(\mathrm{ml})$ de boîtes de prélèvements anciens et récents, essentiellement pour analyses radiocarbone ;

- rapports préliminaires : 2 classeurs ;

- documentation extérieure : tirés-à-part, publications diverses: 1 classeur et $1,5 \mathrm{ml}$ de revues, réparties entre la salle des archives scientifiques et le bureau des enseignants ;

- carnets de fouille ;

- relevés de terrain sur papier millimétré ou calque ;

- relevés finaux sur calque ;

- dessins d'objets sur papier millimétré ou calque ;

- fiches photos ;

- négatifs et positifs argentiques ;

- tirages argentiques ;

- diapositives ;

- photographies numériques ;

- dessins assistés par ordinateur (DAO) ;

- prélèvements.

Ces lots sont stockés dans une étagère à 5 tablettes, soit environ $5 \mathrm{ml}$ de documents, auxquels s'ajoutent 5 tiroirs de plans, ainsi que 3 tiroirs de fiches, négatifs, tirages et diapositives et 3 boîtes de fiches servant d'archivage des supports numériques ( 30 CD-Rom, 2 supports zip ; 2 disques durs externes supplémentaires permettent une copie de sauvegarde de ces données).

Les documents sont majoritairement classés de manière thématique, puis chronologique (ex: dessins 20082009, prélèvements, factures, etc.). Parfois, I'emplacement est opportuniste, en fonction de la place disponible. Le rangement par classeur est ici privilégié, mais les boîtes d'archives, pour les lots plus anciens sont également utilisées.

[1] Cf. orientation bibliographique. 
Le classement est différent dans les tiroirs du meuble à plans. Chaque tiroir possède un lot de documents sur divers supports, mais en relation avec le même secteur. Ainsi, nous retrouvons mêlés tirages photographiques, relevés minute, relevés finaux encrés, plans de synthèse, documents textuels, etc. Ce regroupement et l'agencement

Figure 3

Cahier de fouille du chantier IV de Porsuk, années 1971, 1976 et 1977 (cliché : Soline Morinière / DRAC Alsace)

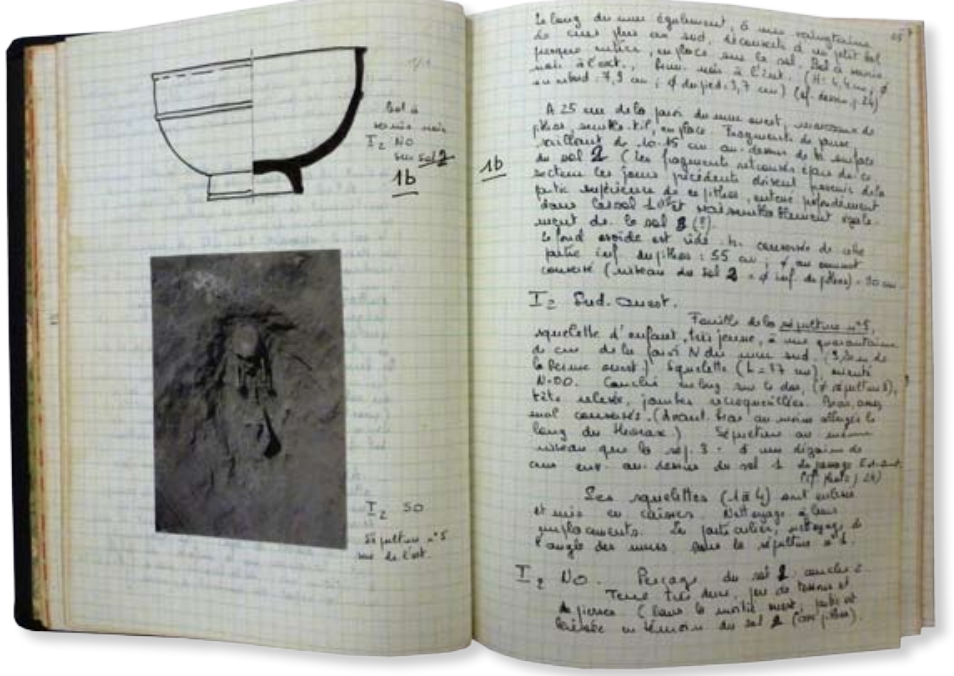

donné aux différentes pièces reflètent un instantané de l'état d'avancement des recherches en cours sur ce secteur. Ainsi, même si l'ordre paraît bien éloigné d'un cadre de classement conventionnel, il convient de respecter celui-ci le temps de la publication, peut-être même de le documenter, car il représente une méthode de travail spécifique d'un architecte dans un cadre géographique et temporel donné.

Grâce à divers contrats, plusieurs campagnes de numérisation de tirages photographiques argentiques ont pu être effectuées, mais la mission n'a pas pu être menée à son terme, faute de crédits alloués. Ceci pose la question de la pérennité de ce genre d'opération, qui plus est dans le cadre d'une interruption de traitement des informations, même si la responsable a noté avec un soin particulier l'état d'avancement du projet sur les tiroirs de documents, selon trois états : «fait», «documents vérifiés, restent les tiff à transformer en jpg» et «matériel dont il n'existe pas de version numérique».

Parallèlement, des plans sur support papier ont été retravaillés en DAO par Françoise Laroche-Traunecker, en fonction des besoins de l'équipe.

Figure 4

Tiroir contenant les documents relatifs au chantier IV de Porsuk (cliché : Soline Morinière / DRAC Alsace)

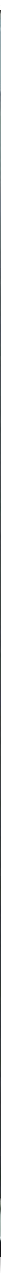




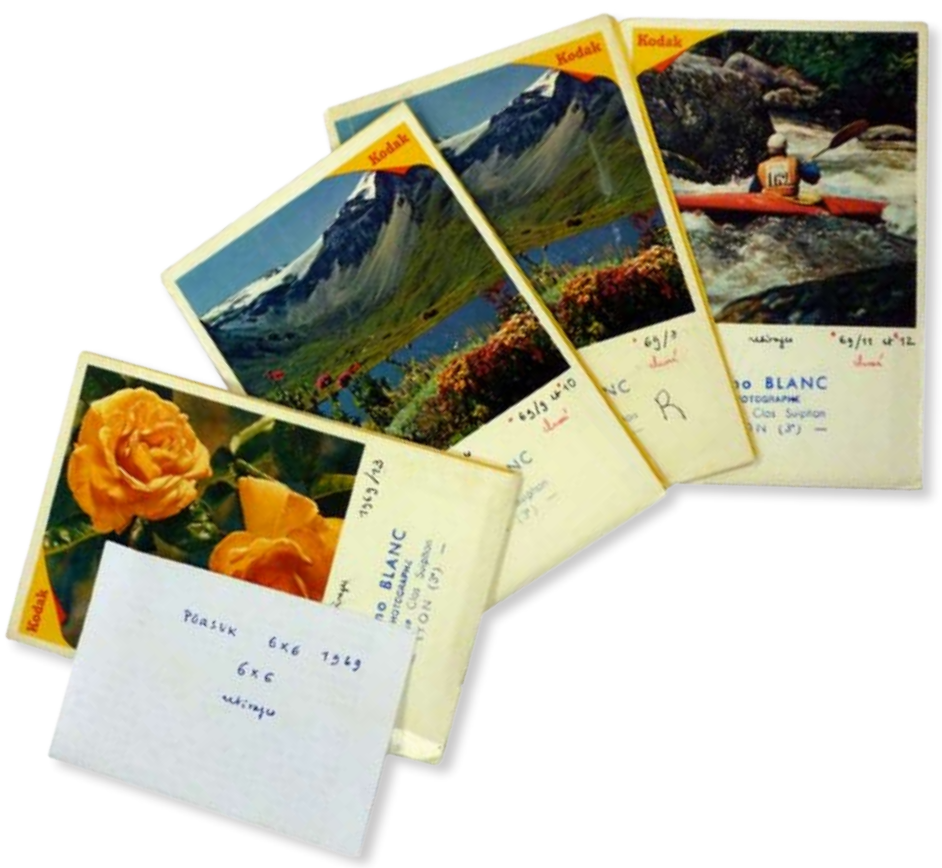

Figure 5

Sélection de tirages photographiques argentiques de la mission de 1969 ayant bénéficié d'une numérisation (cliché : Soline Morinière / DRAC Alsace)

\subsection{TELL HARIRI / MARI : UN FONDS D'ARCHIVES ÉCLATÉ SUR PLUSIEURS UNITÉS DE RECHERCHE [2]}

Tell Hariri / Mari est un des sites urbains les plus importants de Syrie, fondé sur un plan circulaire vers 2900 sur le Moyen-Euphrate, non loin de l'actuelle frontière syro-irakienne et détruit, pour l'essentiel, par les hommes de Hammurabi de Babylone vers 1760 av. J.-C. Mari est fouillée dès 1933 et jusqu'en 1974 par André Parrot, avec une interruption pendant la seconde guerre mondiale. Jean-Claude Margueron, alors enseignant d'archéologie orientale à I'Université de Strasbourg depuis 1969, qui avait un temps travaillé avec André Parrot, prend la direction des fouilles de Mari en 1979. Il récupère ses archives en totalité, qu'il transfère à l'Institut d'archéologie orientale situé alors à l'étage du Palais universitaire de Strasbourg. En 1987, Jean-Claude Margueron, retenu à Paris par sa chaire à l'École pratique des hautes études (EPHE), est remplacé à l'institut strasbourgeois par Önhan Tunca jusqu'en 1990, date à laquelle la chaire d'archéologie orientale sera reprise par Dominique Beyer. Les archives de Mari resteront à Strasbourg jusqu'au milieu des années 1990, puis seront déplacées à Paris. Depuis 2005, la direction des fouilles est assurée par Pascal Butterlin (Université Paris I-Panthéon Sorbonne, UMR 7041 ArScAn). L'ensemble des archives de ce site est à présent conservé au sein de la MAE René Ginouvès à Nanterre, dont le service des archives scientifiques réalise actuellement I'inventaire et la numérisation du fonds.
Pourtant, des axes de recherches liés aux fouilles de Mari sont toujours suivis au sein de I'UMR 7044, notamment la publication des sceaux et des terres cuites mis au jour, ainsi qu'une partie des temples. À l'inverse, des membres de I'UMR participent également à la publication des fouilles récentes et à des programmes parisiens en rapport avec ce site archéologique.

En conséquence, il a été décidé de dupliquer les carnets de fouille de certains secteurs. Ainsi, les photocopies de carnets des campagnes de 1982 à 2004 occupent environ 2,5 ml d'une étagère. En 2005, Pascal Butterlin projette de passer au carnet de fouille numérique : les données de terrain sont saisies au fur et à mesure dans un logiciel de traitement de texte. Ce changement de pratique explique le fait que les photocopies aient été stoppées à cette date.

Ces duplicata représentent la majorité de la documentation encore entreposée à Strasbourg. Parallèlement, quelques copies réduites de plans anciens de la période Parrot, des planches contact, sont stockées dans 4 tiroirs d'un meuble à plan.

Enfin, des CD-Rom contenant une sélection de photos numériques, copiées en fonction des besoins des chercheurs, sont rangés dans une étagère.

Il est toutefois intéressant de noter la présence de nombreux modèles de fiches descriptives d'unités stratigraphiques et d'objets, qu'il pourrait être utile de répertorier afin de documenter I'histoire des techniques d'enregistrement.

[2] Les archives de Mari ne seront traitées ici que dans leur rapport avec I'Université de Strasbourg.

Figure 6

Documentation en rapport avec les fouilles de Mari (cliché : Soline Morinière / DRAC Alsace)

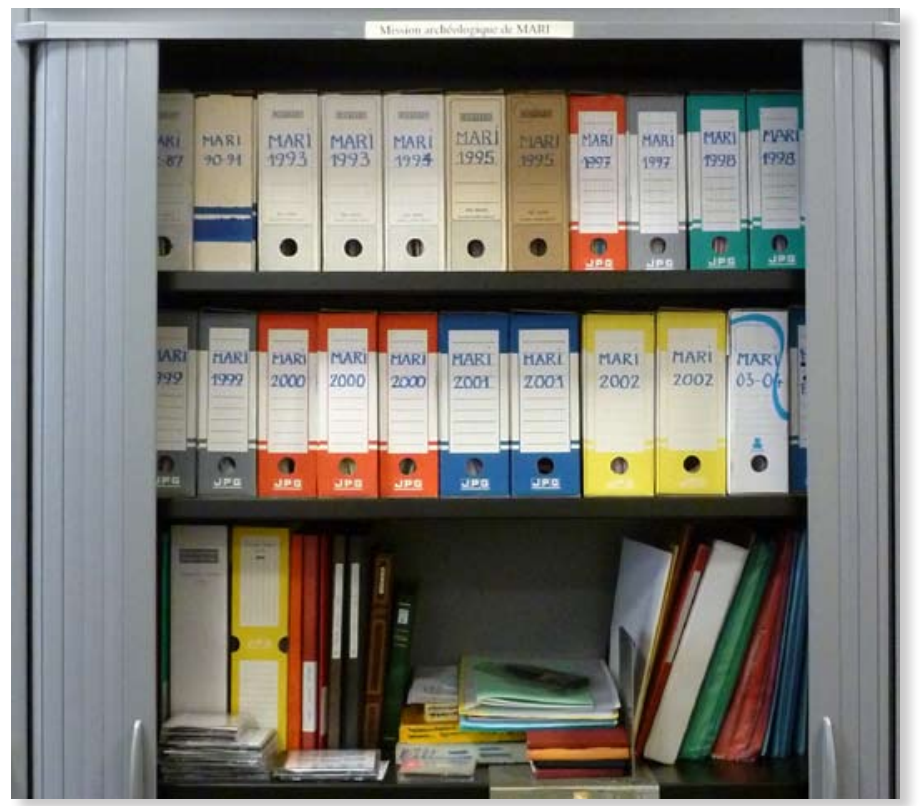




\subsection{RAMADI ET TELL MASHNAQA (SYRIE) : DES MISSIONS ACHEVÉES EN COURS DE PUBLICATION}

\subsubsection{Ramadi}

Ramadi est un site du Moyen-Euphrate syrien, proche de Mari, où ont eu lieu sous la direction de Dominique Beyer deux campagnes de sauvetage (1990-1991) en raison de la menace du passage d'une ligne de chemin de fer. L'origine du site remonte à l'époque d'Obeid (vers 4000 av. J.-C.), mais les vestiges importants appartiennent à la période d'Uruk récent ( $2^{\mathrm{e}}$ moitié $d u$ IVe millénaire) puis au Bronze moyen (début $\mathrm{II}^{\mathrm{e}}$ millénaire).

La série, bien que complète, n'est par conséquent pas volumineuse: il y a 5 boîtes d'archives, contenant essentiellement les cahiers de fouille. Une boîte ancienne renferme des carnets photos et les souches des fiches objet. Enfin, les fiches photos et les tirages occupent un tiroir.

\subsubsection{Tell Mashnaqa}

Tell Mashnaqa est un site de la moyenne vallée du Khabur, occupé du Ve au III e millénaire. Après deux campagnes de fouille (1985-1986) réalisées par JeanYves Monchambert, Dominique Beyer reprend la direction de la mission de 1992 à 2000 pour les phases post-Obeid. Parallèlement, de 1990 à 1995, une équipe

Figure 7

Séries de Ramadi, Méskéné / Emar, et une partie de la documentation de Tell Mashnaqa (cliché : Soline Morinière / DRAC Alsace)

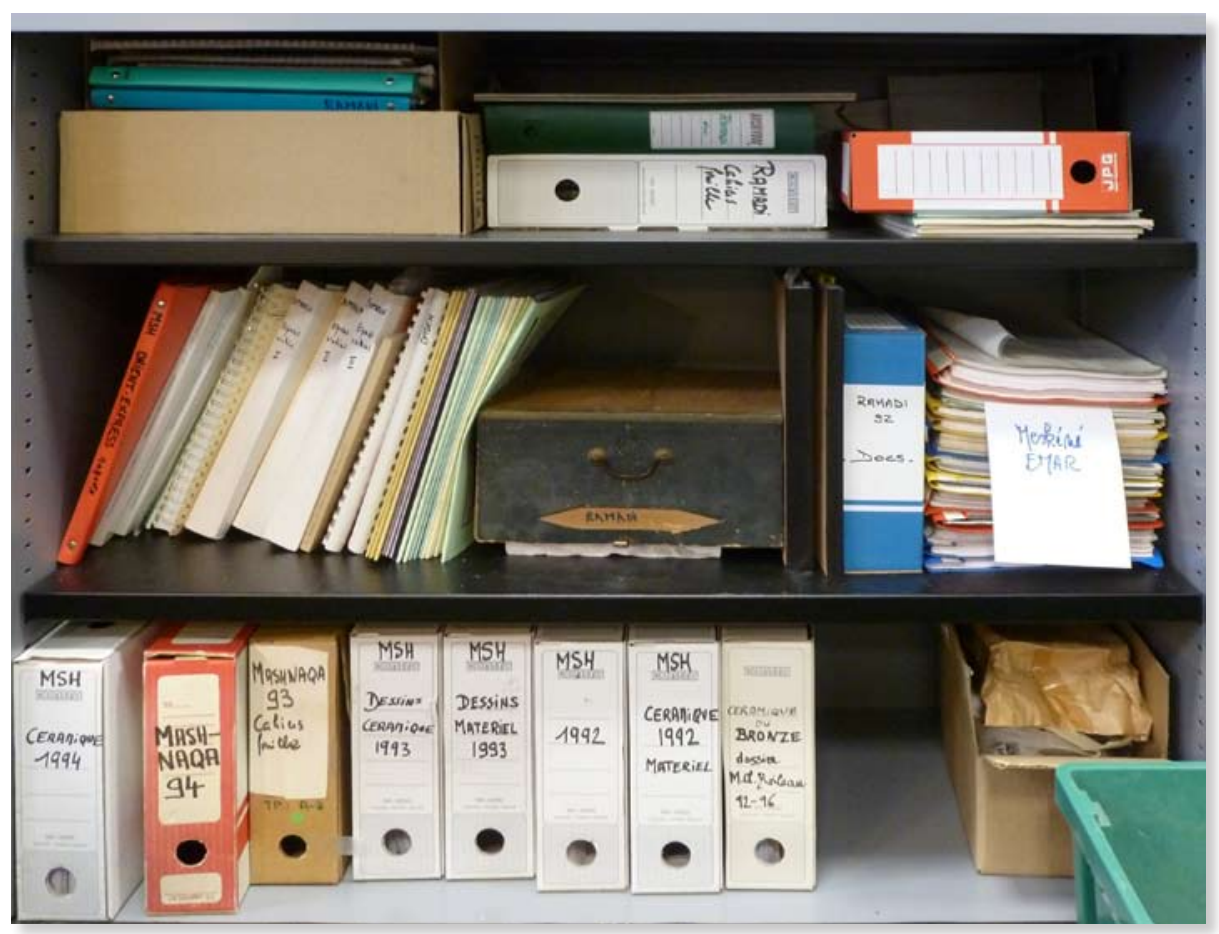

danoise dégage les niveaux anciens. Le résultat des campagnes dirigées par Dominique Beyer est en cours de publication dans le programme Territoires et empires d'Orient, axe Les sites et leur environnement, opération La Syrie du Nord de I'Euphrate au Khabur, du IVe au II millénaire [3].

Ce site offre donc la double particularité d'avoir été fouillé en deux temps après une interruption de six ans et par des équipes de nationalités différentes durant une même période. Les équipes danoise et française ont poursuivi leurs recherches de manière autonome. Mentionnons également le fait qu'une étude du mobilier céramique avait été effectuée sur place dans le cadre d'un mémoire de maîtrise par Valérie Bader à la fin des années 1990. Ces documents seraient actuellement encore partiellement stockés à I'Institut français du Proche-Orient (IFPO) à Damas.

La documentation entreposée dans les étagères occupe environ $4,5 \mathrm{ml}$ de tablettes et 47 contenants. Les archives sont conditionnées dans des boîtes. On y retrouve les grandes catégories déjà décrites :

[3] Cf. orientation bibliographique. 


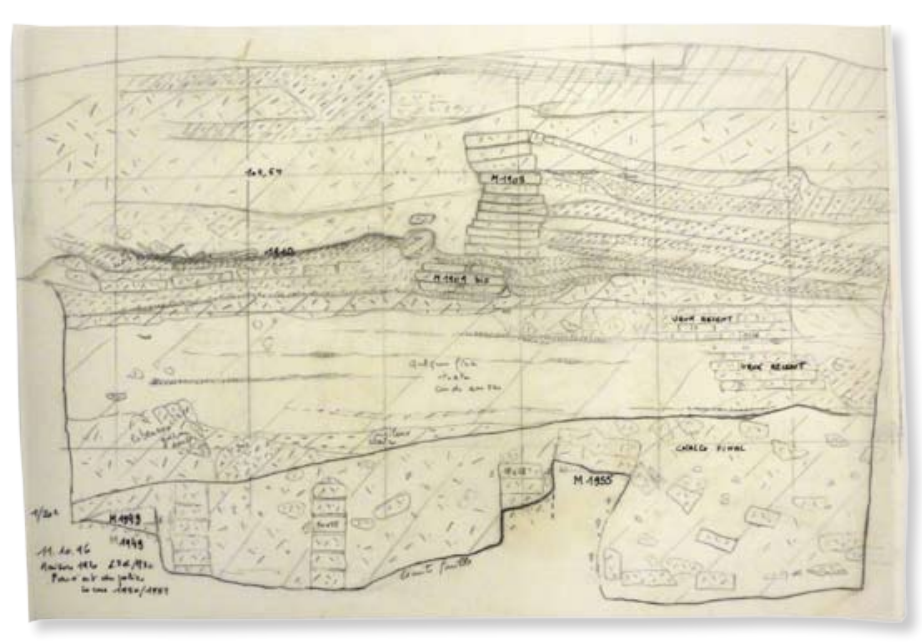

Figure 9

Relevé de la paroi est du locus 1950 / 1951, carrés L3d / L3c à Tell Mashnaqa, 1996 (auteur : Dominique Beyer, cliché : Soline Morinière / DRAC Alsace)

- cahiers de fouille : 5 boîtes ;

- dessins de céramique et petit mobilier : 12 boîtes ;

- topographie : 1 boîte ;

- plans schématiques : 4 boîtes ;

- relevés de parois : 1 boîte ;

- carnets à souche de fiches objet et carnets photo : 2 boîtes ;

- négatifs : 3 classeurs ;

- diapositives : 10 boîtes ;

- documentation diverse ou classée par année : 5 boîtes ;

- rapports préliminaires : 4 boîtes.

Par ailleurs, on compte 3 tiroirs de meuble à plans et plusieurs cases d'un carton à plans. Les fiches objets, les fiches photos et les tirages argentiques sont rangés dans 5 tiroirs.

\subsection{SYRIE DU NORD, MASSIF CALCAIRE [4]}

La Mission archéologique syro-française de la Syrie du Nord, fondée par Henri Seyrig en 1934, a été reprise par Georges Tate (Université de Versailles-Saint-Quentinen-Yvelines) dans les années 80. Depuis 2009, sa direction est conjointement assurée par Gérard Charpentier (Maison de I'Orient et de la Méditerranée / MOM, USR 3439, Lyon) et Maamoun Abdulkarim (Université de Damas et Direction générale des antiquités et des musées de Syrie). Les travaux entrepris par la Mission ne portent pas sur un site isolé mais sur l'ensemble d'une région rurale de la fin de I'Antiquité. Le territoire étudié correspond à une succession de plateaux calcaires situés entre les vallées de I'Afrin de I'Oronte à I'ouest et la plaine de Chalcis à l'est et qui mène, sur

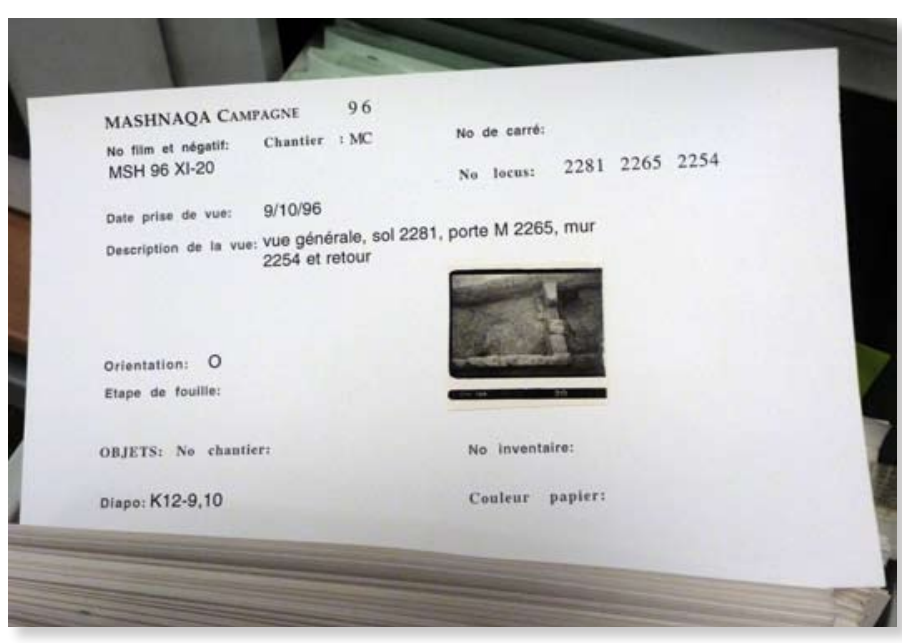

Figure 10

Fiche photo de la campagne 1996 à Tell Mashnaqa (cliché : Soline Morinière / DRAC Alsace)

une centaine de kilomètres, de la frontière turque au nord à la ville d'Apamée au sud. Parallèlement à des études thématiques qui s'appuient sur des prospections menées à l'échelle de la région, la Mission a entrepris des opérations de fouilles sur quatre sites de référence : les villages de Serğilla, d'El Bāra et de Ruweiha, dans le ğebel Zawiyé au sud, et le village de Déhès, dans le ğebel Barisha au nord.

Nous sommes dans le cas d'une mission archéologique multi-institutionnelle, à laquelle collaborent des membres de I'UMR 7044 et les archives de la Mission sont gérées à partir de plusieurs sites. L'Institut français du ProcheOrient (IFPO) a conservé à Damas les fonds Seyrig et Tchalenko (avant 1980) et une partie des archives Tate (1980-1990). En 2009, la MOM de Lyon a accueilli I'ensemble de la documentation de terrain produite par les fouilles de Serğilla, Ruweiha et El Bâra (1988-2011) ; elle a également accepté la charge d'un important fonds cartographique et aéro-photographique produit dans les années 1940-1950. Le Laboratoire Archimède (UMR 7044) est quant à lui dépositaire :

- de la documentation produite lors de la fouille du village de Déhès dirigée par Bernard Bavant des années 1990 aux années 2000 ;

- des archives numériques (photos, documentation figurée, analyses, etc.) issues de l'étude de l'architecture domestique menée depuis les années 2000 sur plusieurs sites du ğebel Zawiyé par Catherine Duvette.

Les publications de ces travaux sont intégrées aux programmes de recherches de l'équipe « Territoires et empires

[4] Catherine Duvette est I'auteur de cette description : nous la remercions pour sa participation. 
Dessins d'objets mis au jour lors des fouilles du village de Déhès (Fr. Mercier)
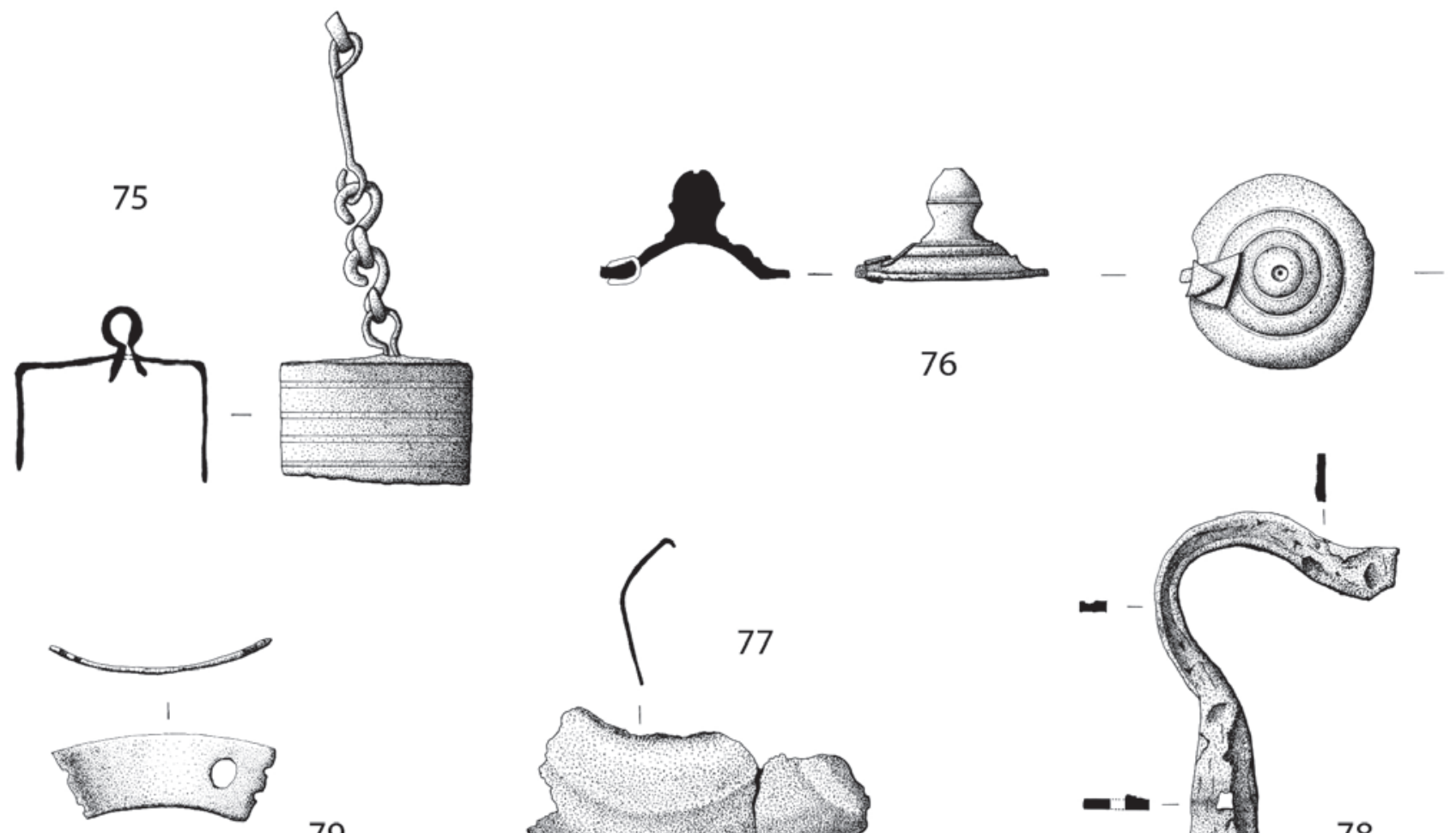

79
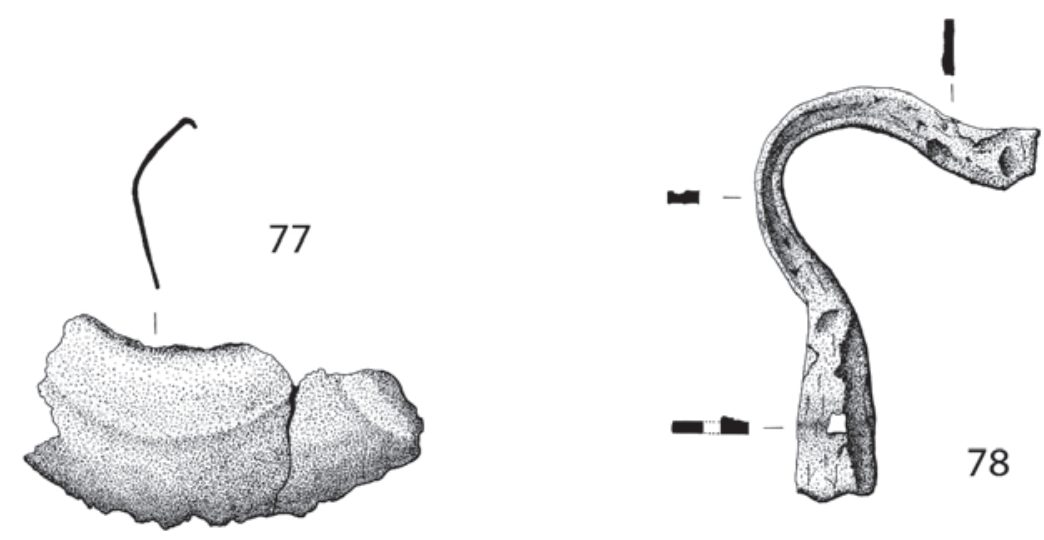

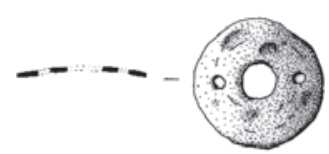

80

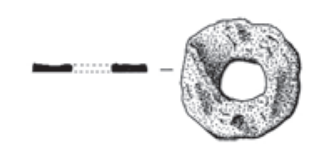

81

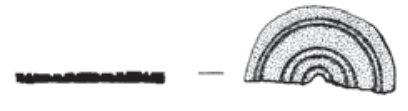

82
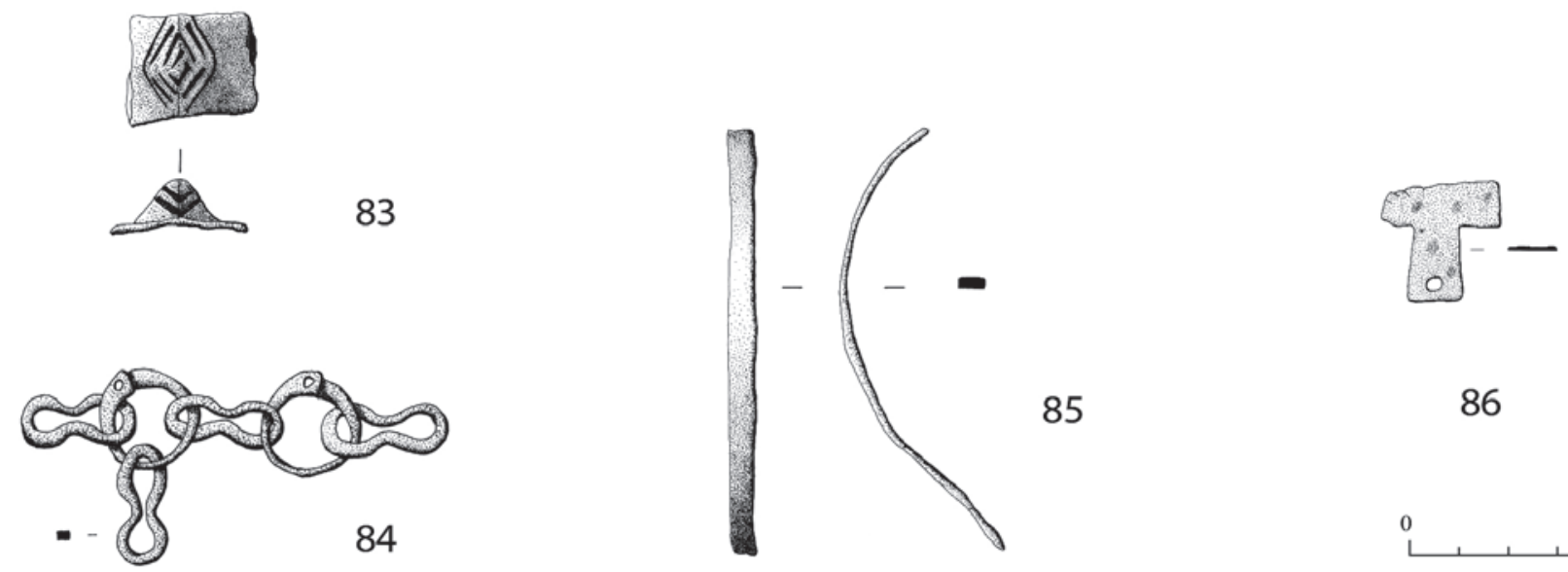

86

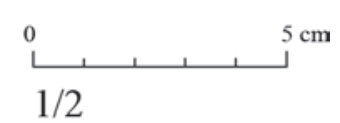




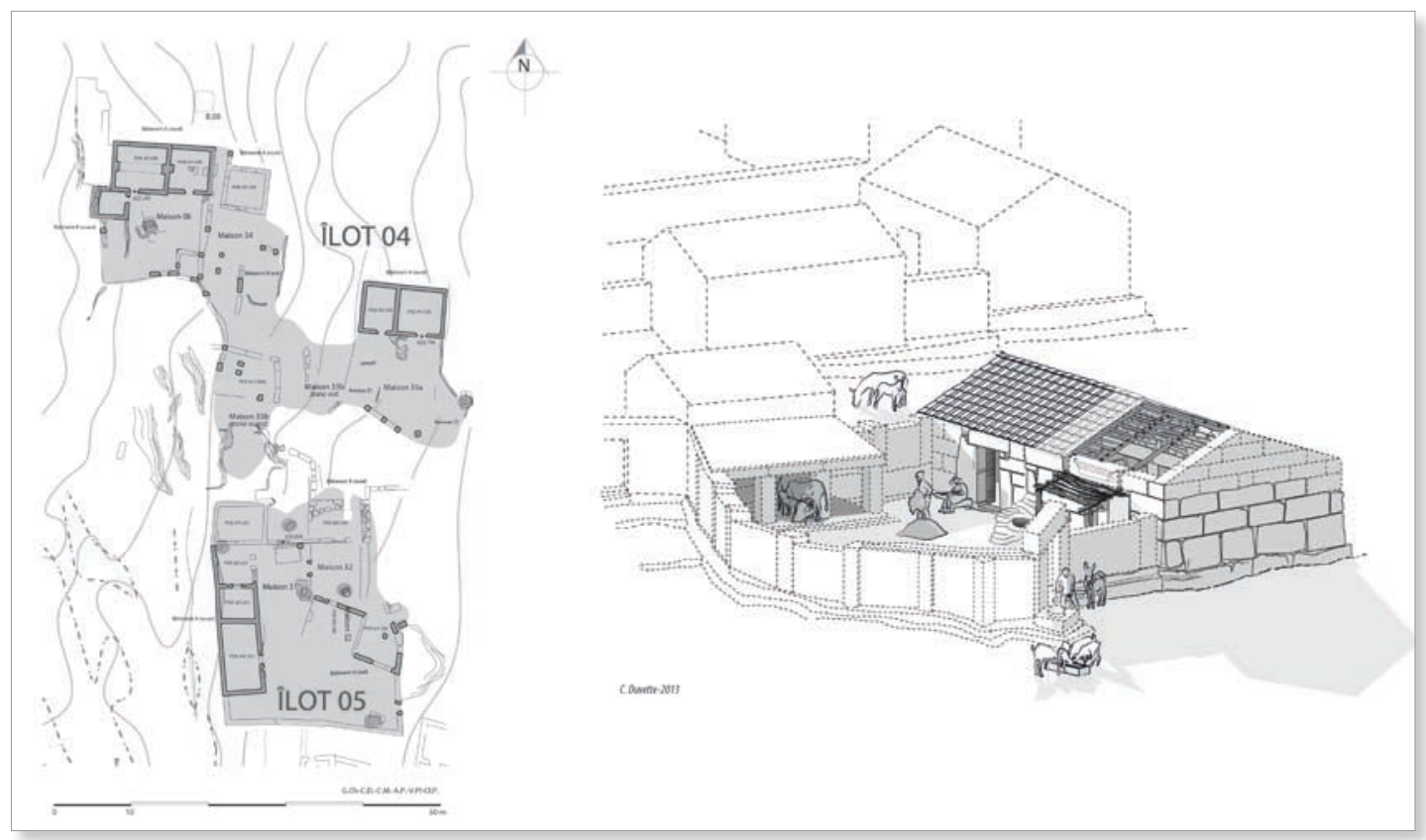

Figure 12

Étude d'une maison du village de Serğilla (C. Duvette)

\section{Figure 13}

Modèle numérique de terrain, le Massif calcaire de Syrie du Nord (J.-Ph. Droux)

d'Orient », axe « Les sites et leur environnement », opération « Les Balkans, Chypre et la Syrie du Nord $\mathrm{d}^{\prime}$ Apamée à la frontière turque, du IV $\mathrm{V}^{\mathrm{e}}$ au $X \mathrm{~V}^{\mathrm{e}}$ siècle ». Les archives physiques afférentes à l'étude du village de Déhès sont entreposées sur une étagère d'environ $5 \mathrm{ml}$ et dans 4 tiroirs de meuble à plans. Elles sont constituées de fiches, de photos argentiques, de minutes de terrain et de planches de dessins sur calque végétal et papier normal ou millimétré. Ces documents sont de formats divers (du A4 au A0). Ils concernent les études stratigraphiques et architecturales du village et celles de diverses catégories de mobilier (objets, verres, céramiques, etc.). Une partie du fonds a été numérisée en 2013 et 2014 et le reste de la documentation est en cours de traitement. Les archives numériques afférentes à la fois à la publication de Déhès et à l'étude de l'architecture domestique sont quant à elles stockées sur plusieurs disques durs appartenant au Service d'analyse des formes architecturales et spatiales du Laboratoire Archimède. Les formats de ces documents numériques sont divers et en constante mutation. Rares sont ceux qui pourraient, à ce jour, être qualifiés de «dormants ».

Afin de garantir leur pérennité, les trois institutions concernées par les archives de la Mission archéologique syro-française de Syrie du Nord (IFPO, MOM et Archimède) travaillent à leur harmonisation et à leur

[5] https://halshs.archives-ouvertes.fr

[6] https://medihal.archives-ouvertes.fr

[7] www.archeorient.mom.fr/recherche-et-activites/ participation-a-reseaux/lyon2-2013-geolyon

[8] Afin de développer ce projet, nous nous basons sur I'expérience du programme ArkeoGIS (http://arkeogis.org.

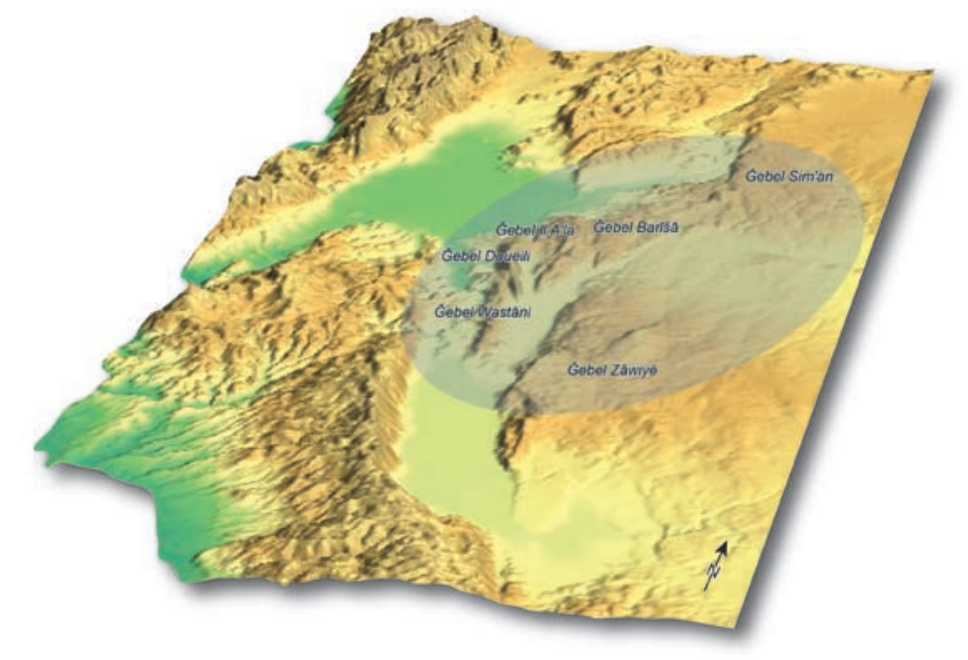

partage à partir de plusieurs plateformes institutionnelles : le portail HAL-SHS [5] pour la documentation publiée et non publiée, le portail MédiHAL [6] pour les photothèques et les données figurées et enfin, le portail GéoLyon [7] pour les collections cartographiques et aéro-photographiques. Ce programme de partage s'inscrit dans un projet propre à la Mission qui vise à mettre en place un agrégateur de bases de données, géographiques, topographiques, archéologiques et patrimoniales, qui permettrait à l'ensemble de la communauté scientifique qui travaille sur la région de pouvoir simultanément géolocaliser les sites étudiés et avoir une vision synthétique de la documentation scientifique qui en fait mention. Ce projet [8] est actuellement en cours d'élaboration par les Services de techniques archéologiques de la MOM (Gérard Charpentier et Alexia Levray) et le Service d'analyse des formes architecturales et spatiales du Laboratoire Archimède (Jean-Philippe Droux et Catherine Duvette). 


\subsection{MESKÉNÉ / EMAR}

Mékéné / Emar est situé sur le Moyen-Euphrate syrien, à la hauteur de la ville d'Alep. Le site renferme les vestiges de la ville ancienne d'Emar, connue par les textes de Mari dès le IIIe millénaire, mais semble-t-il rebâtie par le conquérant hittite Suppiluliuma dans la seconde moitié du XIVe s. av. J.-C. Elle est détruite autour de 1180 av. J.-C., au moment où s'effondrent tous les grands centres urbains du Bronze récent. Une fouille de sauvetage a été réalisée de 1972 à 1976 sous la direction de Jean-Claude Margueron avec une équipe d'étudiants strasbourgeois pour l'essentiel.

La série n'est composée que de photocopies de cahiers de fouille rangées dans des dossiers sur 0,30 ml d'une tablette d'étagère.

Les dessins et photographies de sceaux sont conservés par Dominique Beyer, en charge de la publication de la collection [9].

\subsection{LYCIE (TURQUIE) [10] : UNE MISSION ACHEVÉE EN PARTIE PUBLIÉE}

Une prospection relative aux villes de Lycie a été réalisée sous la direction d'Edmond Frézouls, professeur d'histoire romaine à I'Université de Strasbourg. Deux cités ont été étudiées : Sidyma et Kadyanda, durant trois campagnes au cours des années 1980 (1982, 1984, 1986). Une partie des résultats a été publiée dans la revue Ktèma, par Sylvie Dardaine, Edmond Frézouls, Daniel Longepierre, Marie-José Morant, puis par Anne Jacquemin [11].

[9] Cf. orientation bibliographique

[10] Nous tenons ici à remercier chaleureusement MarieJosé Morant qui nous a transmis l'ensemble des informations concernant cette opération.

[11] Cf. orientation bibliographique

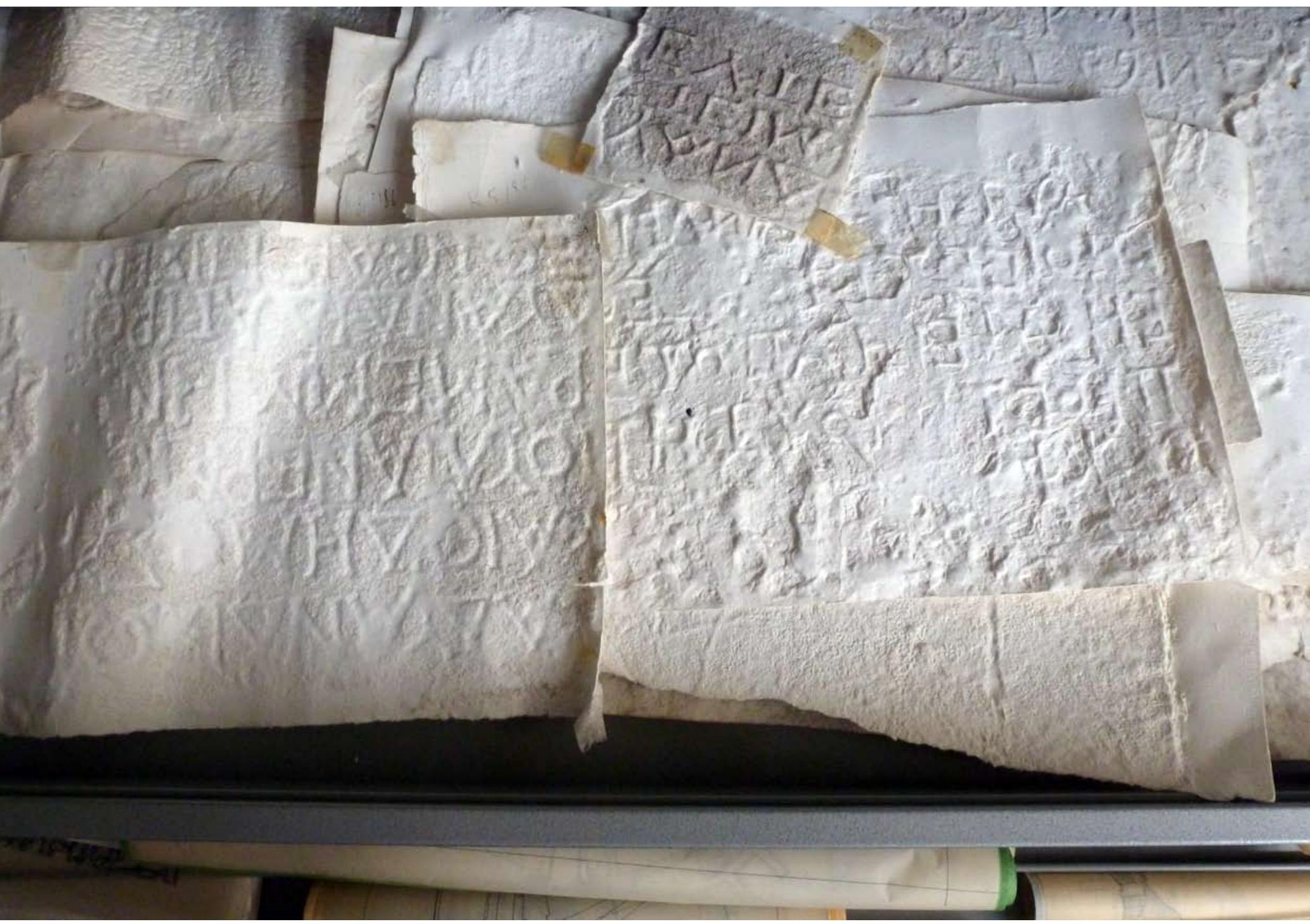


La documentation produite lors de ces missions regroupe :

- des plans et relevés généraux de ces villes et des tombes, réalisés par l'architecte Daniel Longepierre (un tiroir de meuble à plans) ;

- des prises de vues : négatifs, tirages argentiques et diapositives,

- et des notes de terrain, dont I'ensemble représente environ $1 \mathrm{ml}$.

À ces catégories communes, que I'on retrouve présentes dans les fonds précédemment décrits, s'ajoute une série originale et volumineuse d'estampages d'inscriptions grecques. Ceux-ci, réalisés sur papier buvard blanc après humidification puis séchage, occupent 2 tiroirs de meuble à plans.

\subsection{GÜLNAR (TURQUIE) : UN CAS D'ARCHIVES HÉRITÉES [12]}

Le site archéologique de Meydancikkale, en Anatolie méridionale, a été fouillé et étudié sous la direction d'Emmanuel Laroche durant de nombreuses campagnes allant de 1971 à 1982. Les vestiges mis au jour pourraient remonter à l'époque louvite mais datent principalement des époques perse et hellénistique, puis d'une dernière occupation byzantine.

Il s'agit d'une fouille achevée depuis plusieurs années, dont la documentation a été entreposée à la MISHA par Françoise Laroche-Traunecker, fille du directeur de la mission, dans l'optique d'une conservation au sein d'une structure de recherche. Un inventaire sommaire a été effectué par ses soins dans le cadre de l'enquête Archives de la recherche en sciences humaines et sociales (ARSHS) réalisée en 2004-2008 [13]. Une publication a été éditée en 1998 [14].

Le lot d'archives entreposé à la MISHA occupe 2,4 ml dans une étagère et est composé de 18 boîtes de diapositives, et d'une tablette de dossiers, principalement de la correspondance, des relevés architecturaux et des tirages photographiques. Il n'est pas complet. Selon Françoise Laroche-Traunecker, les documents publiés (calques de dessins, photos) sont restés chez l'éditeur. Beaucoup de documents inédits sont conservés par Alain Davesne, directeur de la mission de Gülnar à partir de 1985 .

Cette documentation ne semble pas intégrée dans un

[12] Informations recueillies directement auprès de Françoise Laroche-Traunecker, que nous remercions pour son aide.

[13] Cet inventaire ne semble toutefois pas apparaître sur le site PANDOR de la MSH de Dijon, dont un instrument de recherche répertorie les résultats de l'enquête. Pour plus d'informations sur cette enquête, Cf. WOLIKow 2009

[14] Cf. orientation bibliographique axe de recherche en cours. Une description exhaustive de cette série et une diffusion de l'information pourraient s'avérer très bénéfiques à une valorisation ultérieure des données récoltées.

\subsection{LE FONDS JACQUELINE PIRENNE : DES ARCHIVES SANS SUCCESSEUR [15]}

Jacqueline Pirenne, épigraphiste et archéologue du Sud arabique, s'est vu proposer un séminaire d'études sabéennes à l'Université de Strasbourg en 1987, et transfère les archives de ses travaux et prospections à I'Institut d'archéologie classique du Palais universitaire [16]. Elle décède brutalement en 1990, et aucun successeur ne reprendra le séminaire.

Au début des années 1990, Chaker Ghadban, alors enseignant dépendant de l'Institut d'archéologie classique, a été chargé d'exploiter la documentation, mais ce travail n'a pas abouti. Edmond Lévy, alors doyen de la Faculté des sciences historiques, a par la suite accepté de transférer certains documents au Cabinet des études sémitiques du Collège de France. En 2006, Denyse Vaillancourt, ingénieur CNRS à I'UMR 7044, puis André Pautler, également ingénieur CNRS de I'UMR 7044, ont entrepris le catalogage de la bibliothèque (environ 900 publications) sur une base Filemaker versée dans le Catalogue collectif inversé du réseau Frantiq Fédération et Ressources sur I'Antiquité. Suite au déménagement des collections à la MISHA, le fonds imposant a rejoint le local d'archives de I'UMR dans trois armoires. La majorité des ouvrages de la bibliothèque personnelle de Jacqueline Pirenne a intégré la bibliothèque de la MISHA.

Ce fonds, tout comme celui d'Emmanuel Laroche pour Gülnar, a fait I'objet d'un inventaire par Françoise Laroche-Traunecker en 2008, lors de I'enquête ARSHS (Cf. supra), inventaire qui sera repris dans le descriptif ci-dessous [17].

Il s'agit là d'un des rares fonds qui porte aussi fortement l'empreinte personnelle du travail d'un chercheur d'un bout à l'autre de la chaîne opératoire. Nous y trouvons les sources primaires, les travaux de synthèse en vue de publications, les ouvrages eux-mêmes, et des archives annexes en lien avec les activités de recherche. Les dates extrêmes vont de 1950 à 1985.

[15] La majorité des informations a été obtenue de Françoise Laroche-Traunecker, que nous remercions pour son aide précieuse.

[16] LORENTZ 2000, p. 20.

[17] Comme dans le cas de Gülnar, cette collection ne figure pas dans la nouvelle version de l'instrument de recherche consacré à ARSHS. 


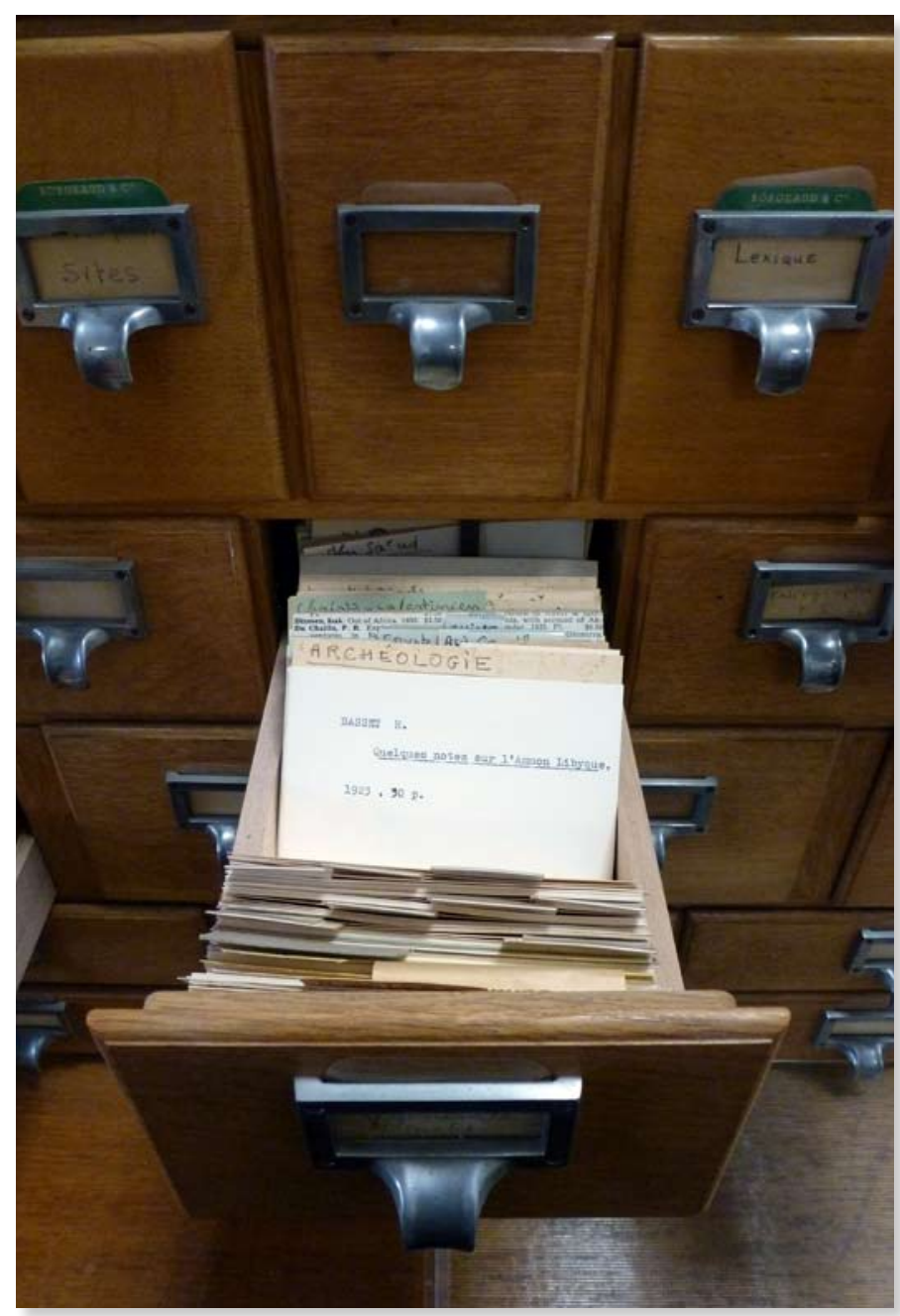

Figure 15

Fichier bibliographique du fonds Jacqueline Pirenne (cliché : Soline Morinière / DRAC Alsace)

Ont été répertoriés dans les deux étagères dédiées à cette collection :

- 2 cartons de photographies et diapositives ( $1 \mathrm{ml}$ ) ;

- 1 carton de posters ( $1 \mathrm{ml}$ ) ;

- une vingtaine de classeurs relatifs aux travaux de terrain (fouilles, épigraphie, ethnographie) $(1,5 \mathrm{ml})$;

- rapports, tirés-à-part, publications diverses (env. $1 \mathrm{ml}$ et un carton) ;

- une tablette d'artefacts, essentiellement des prélèvements, dont certains échantillons botaniques $(1,2 \mathrm{ml})$;

- une collection de fiches thématiques réparties dans une dizaine de boîtes et hors contenant $(1,5 \mathrm{ml})$.

À cela s'ajoute un meuble à tiroirs, dont le niveau inférieur possède 4 tiroirs de plans et relevés et 2 tiroirs de documents divers; la partie supérieure est composée de 23 tiroirs de fiches thématiques très variées (lexique, sites, bibliographie, paléographie, etc.) et de 6 tiroirs de classeurs de négatifs et diapositives. Un carton de déménagement, contenant essentiellement de la documentation publiée, vient compléter cet ensemble.
Il s'agit, semble-t-il, de la collection la plus importante en terme de volume, et qui renferme un potentiel inexploité. En effet, Jacqueline Pirenne [18] fait partie des pionniers de la recherche sur le territoire Sud arabique. De plus, ses travaux dépassent les limites des fouilles archéologiques : tout à la fois épigraphiste et archéologue, elle a également rassemblé des données et artefacts en lien avec diverses observations de nature ethnographique. Notons en outre la présence de documents rédigés par André Maricq [19], qui a traduit pour elle des sources grecques en vue de la publication du Royaume sud-arabe de Qataban et a collaboré à la datation du Périple de la mer Erythrée. L'exploitation du contenu scientifique, dont certaines informations ne sont peut-être pas publiées, ainsi qu'un inventaire exhaustif des documents contribueraient à valoriser ce fonds et à mieux faire connaître le travail de ce chercheur.

\subsection{LES COLLECTIONS PÉDADOGIQUES}

Parallèlement aux fouilles archéologiques sur le terrain, les activités d'enseignement font partie intégrante des missions de certains membres de I'UMR. À ce titre, elles doivent être prises en compte lors d'une opération d'inventaire des archives de la discipline dans un établissement d'enseignement supérieur et de recherche.

\subsubsection{Les plaques de verre et les diapositives}

Des supports visuels sont projetés durant les cours magistraux. L'ancienne collection de plaques de verre de I'Institut d'archéologie du Proche-Orient est stockée dans le local d'archives. Les documents sont rangés dans 14 casiers, selon un classement géographique.

Ces plaques ont fait l'objet d'un inventaire à la pièce, qui est consultable en ligne [20].

Une importante collection de diapositives a succédé aux vues sur plaque de verre. Celle-ci est entreposée dans le bureau des enseignants-chercheurs en archéologie orientale, et occupe deux tablettes $(2,4 \mathrm{ml})$ et 6 tiroirs de meuble à dossiers suspendus. 2 types de classement ont été réalisés : thématique et géographique. Une grande partie de ces diapositives figure des reproductions de documents tirés de publications, mais une proportion non négligeable, compte tenu du volume imposant de la

[18] WiLL 1991

[19] SeYRIG 1961.

[20] http://www2i.misha.fr/flora/servlet/LoginServlet 


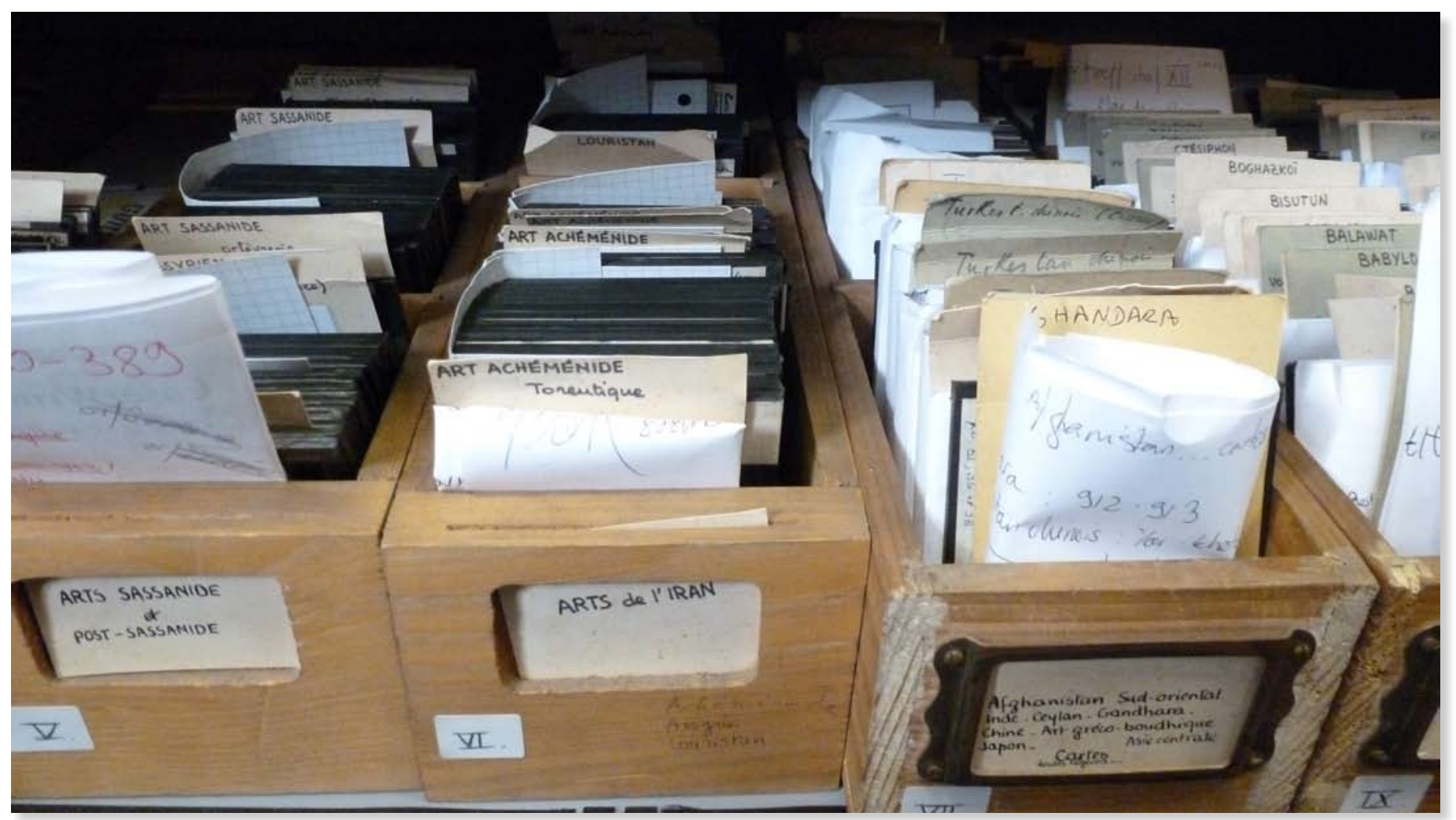

Figure 16

Lot de plaques de verre de l'Institut d'archéologie du Proche-Orient (cliché : Soline Morinière / DRAC Alsace)

collection - plusieurs milliers de pièces -, représente des vues prises sur le terrain.

\subsubsection{Les moulages et empreintes}

Parallèlement aux documents à deux dimensions, des séries de moulages et empreintes constituent les collections d'étude du fonds proche-oriental.

\subsubsection{Les empreintes de sceaux et de scellements de porte}

Ces empreintes, correspondant essentiellement à des sceaux du département des antiquités orientales du Musée du Louvre, sont entreposées dans le bureau des enseignants-chercheurs en archéologie orientale. Un lot représentatif des productions de différentes époques et origines géographiques est conditionné dans un meuble à tiroir, spécialement recyclé pour cet usage.

Une autre série, consacrée exclusivement à Tell Hariri / Mari, est stockée dans 2 boîtes d'archives rigides. En tout, la collection représente plusieurs centaines d'empreintes.

Enfin, un lot conséquent, mais encore à identifier, est stocké dans les réserves du musée des moulages, au Palais universitaire.

[21] Cf. article de Soline Morinière consacré à ce sujet dans le présent numéro.

[22] Michaelis 1897, p. 2-7.

\subsubsection{Les moulages}

La collection des moulages de l'Université de Strasbourg [21] renfermant plus d'un millier de pièces, est une des plus importantes collections universitaires de France. Créée par Adolf Michaelis à partir de 1872, celleci est destinée à montrer chronologiquement l'évolution de l'art grec et romain. Une série dite «assyrienne», représente la première des douze sections. Décrite dans le catalogue de 1897 [22], elle comptait à l'origine près

Figure 17

Lot de diapositives classées par site, destiné aux cours d'archéologie orientale (cliché : Soline Morinière / DRAC Alsace)

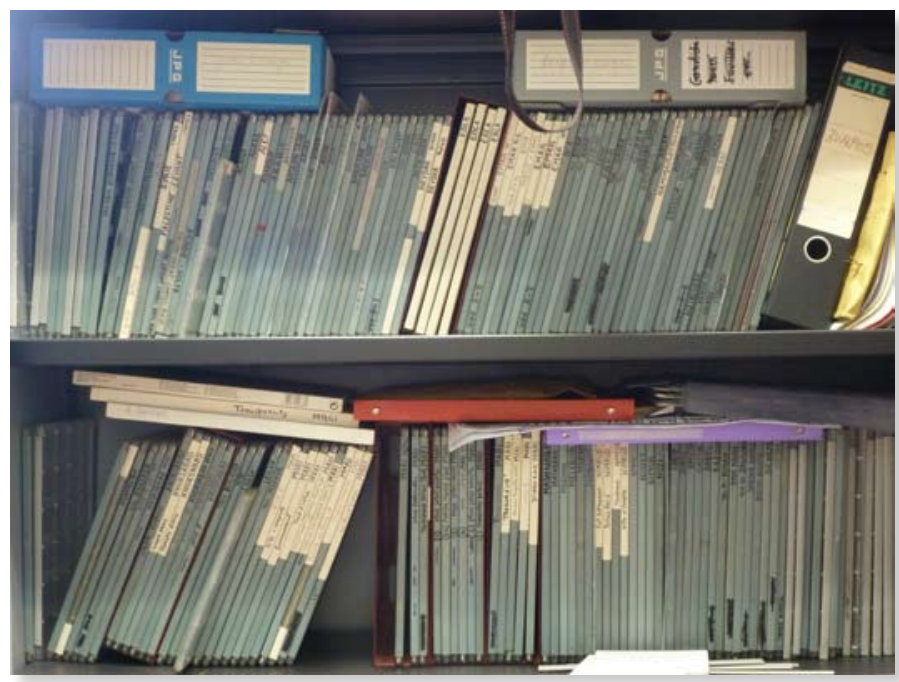




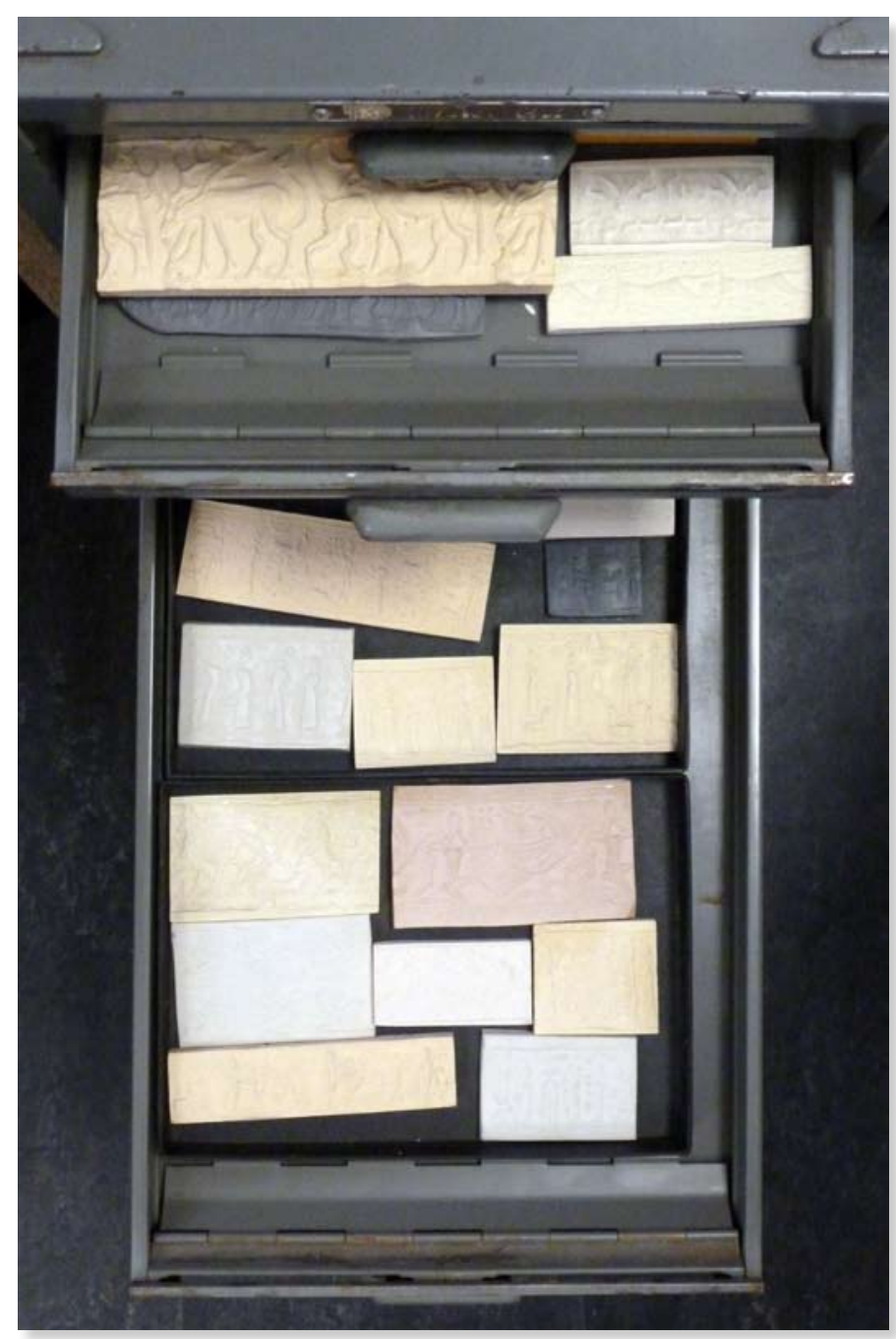

Figure 18

Empreintes de sceaux-cylindres du Proche-Orient ancien (cliché : Soline Morinière / DRAC Alsace)

$d^{\prime}$ une quarantaine de moulages ( $n^{\circ} 7$ à $44,47-48$ ), allant de la statue de Gudea de Tello (Iraq) au relief de Cybèle de Böjuk Suret (Turquie).

\subsection{La question des archives numériques}

Lors de la description de chaque série, nous n'avons que très peu abordé les documents numériques, qu'ils soient natifs ou numérisés. Seuls quelques supports CDRom parsèment ça et là la documentation papier, I'essentiel des fichiers étant stocké actuellement sur les disques durs des ordinateurs des membres de I'UMR, avec sauvegarde effectuée sur plusieurs disques durs externes. Une sauvegarde générale sur un serveur dédié ne semble pas être pratiquée, du moins par les personnes consultées, peut-être parce qu'il s'agit de documents en cours de modification, ou non publiés. Pour certaines opérations, une mission spécifique $d^{\prime}$ 'inventaire et $d$ 'indexation devrait être effectuée sur ces documents, dont la conservation peut poser problème à moyen terme.

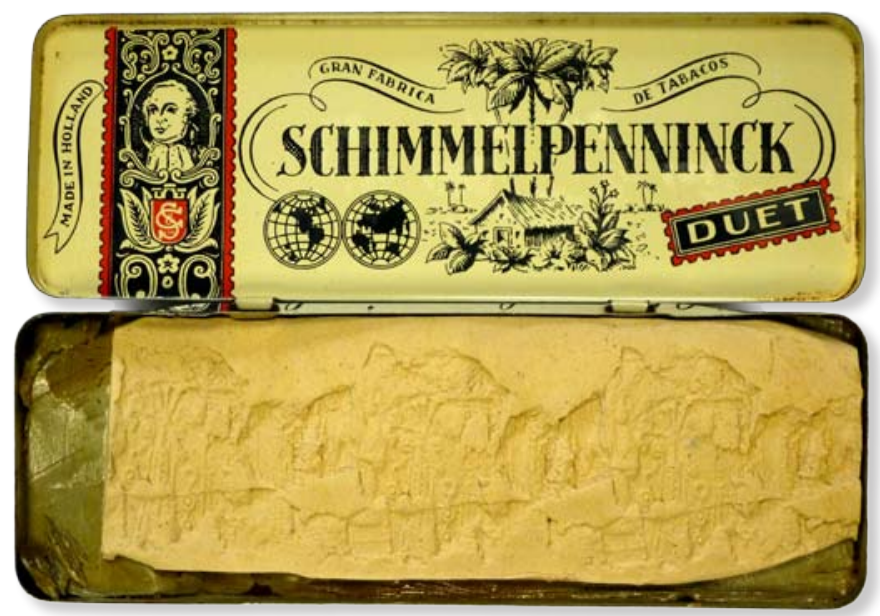

Figure 19

Empreintes de sceaux-cylindres mis au jour sur le site de Tell Hariri / Mari (cliché : Soline Morinière / DRAC Alsace)

La mise en place d'un système proposant la gestion des archives numériques et de leurs métadonnées liées serait à envisager en concertation avec les autres composantes de I'UMR, voire, peut-être au niveau même de la MISHA. Des infrastructures, comme Huma-Num [23] par exemple, apportent leurs compétences et un ensemble de services liés à la conservation et la diffusion des données de la recherche.

\section{LES ARCHIVES DE LA RECHERCHE EN ARCHÉOLOGIE : DES COLLECTIONS À VALORISER}

Cette description sommaire des collections documentaires issues des activités des enseignants et chercheurs en archéologie orientale ne donne qu'un bref aperçu de la diversité et de toute la richesse potentielle que renferme cette série. D'autres composantes existent au sein de I'UMR 7044, dont chacune possède un volume conséquent de données scientifiques et d'archives en relation. Celles-ci doivent avant tout être publiées. Mais le cycle de vie de ces documents ne s'arrête pas là ; en effet, leur prise en charge en tant que fonds d'archives par le biais d'un classement des pièces, d'un inventaire et d'un traitement des fichiers numériques permettrait une diffusion de l'information et une valorisation des données et des documents, profitables aux chercheurs, aux UMR, MSH et universités et, plus largement, à l'histoire de la discipline dans son ensemble.

[23] www.huma-num.fr 


\subsection{AUTORISER UNE (RE)VISITE DES DONNÉES SCIENTIFIQUES}

L'archive archéologique résulte de l'étude d'un fait qui, la plupart du temps, a été détruit pour accéder aux niveaux stratigraphiques inférieurs. En cela, elle est le seul témoin de l'existence passée d'une structure, une couche ou encore un objet. Elle constitue donc une source primaire qu'il convient de conserver, car en un certain sens, elle acquiert un statut d'original : un retour sur le terrain pour une nouvelle observation ne pourra pas être envisagé. La publication des résultats des fouilles met en jeu l'analyse de l'archéologue, qui reprend ces données : les relevés sont retravaillés, les photos recadrées et/ou retouchées, les informations de terrain interprétées afin de donner à la communauté scientifique une synthèse qui représente son cœur de métier.

Le fait d'autoriser a posteriori un accès aux documents

\section{Figure 20}

Meuble du fonds Jacqueline Pirenne, entreposé dans le local archives de la MISHA (cliché : Soline Morinière / DRAC Alsace)

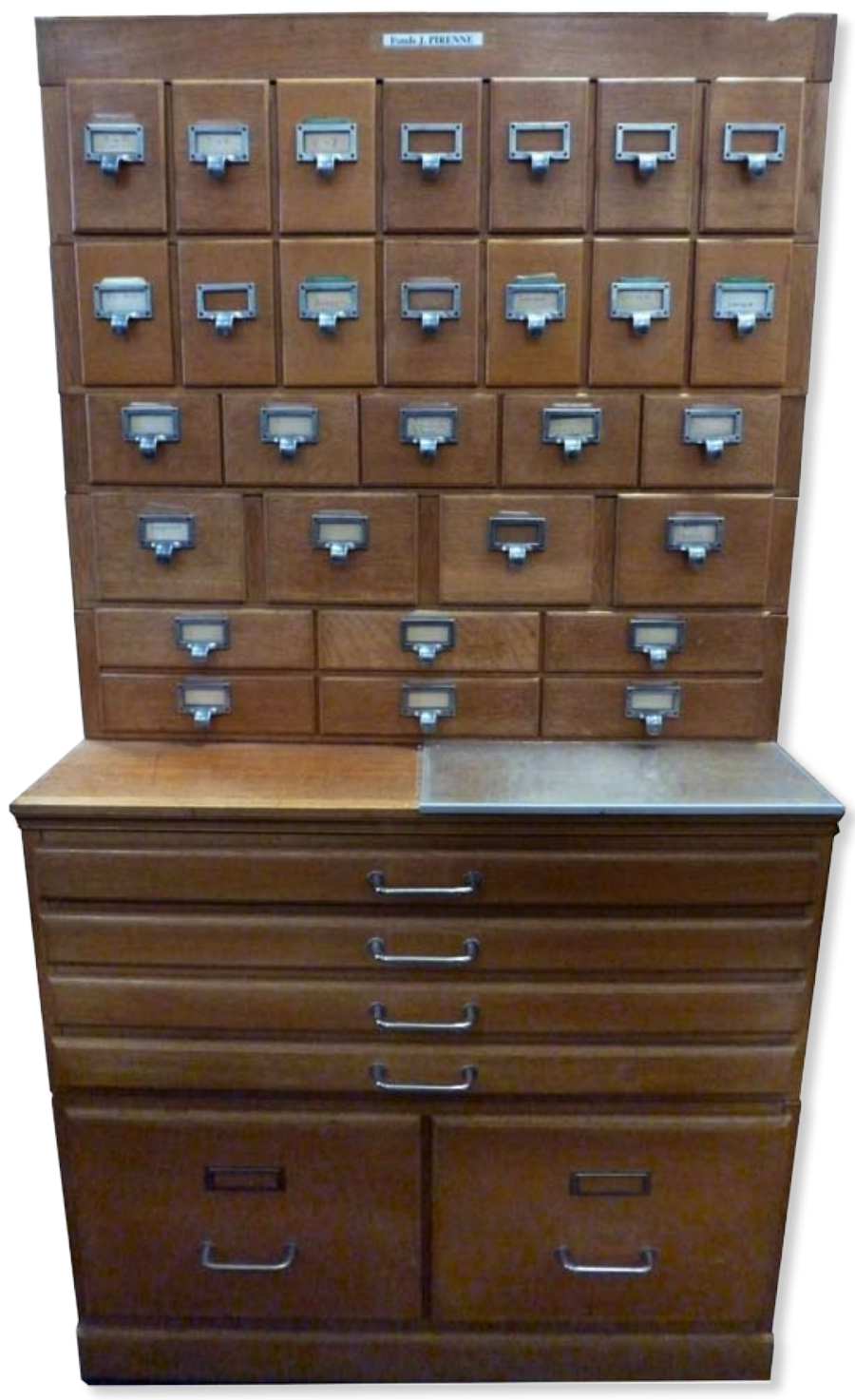

primaires d'une opération archéologique achevée, par I'intermédiaire d'une description archivistique, permet une revisite des données à la lumière des nouvelles découvertes effectuées depuis la publication. La mise en commun des inventaires de plusieurs missions pour une même composante offre aux chercheurs la possibilité d'une recherche thématique qui dépasse le cadre strict de la monographie de site.

De plus, il n'est pas rare que la diffusion des résultats n'aboutisse pas à publication éditée exhaustive. Il est alors d'autant plus important d'effectuer un inventaire de ces documents pour en accroître la visibilité au sein de la communauté scientifique, susceptible d'en exploiter tout ou en partie les données.

\subsection{L'ARCHIVE EN TANT QU'OBJET PATRIMONIAL : UNE PISTE À EXPLOITER}

\subsubsection{Contenus et contenants muséables}

Mais l'archive n'est pas qu'un support de données. D'une part, elle constitue elle-même un objet à valoriser en tant que collection patrimoniale ; d'autre part, les contenants, qu'il conviendrait également de conserver, aident à contextualiser les documents.

\section{Le meuble de Jacqueline Pirenne: un cabinet de curiosités}

Une partie du fonds de Jacqueline Pirenne est rangée dans un meuble spécialement adapté aux documents qu'il contient. Il s'agit d'une bibliothèque à tiroirs, que nous avons brièvement décrite précédemment, et qui renferme l'essentiel de ses recherches, qu'elles soient bibliographiques, épigraphiques ou archéologiques. L'organisation interne de chaque tiroir est propre aux types de documents archivés. Les supports, pour la plupart des fiches cartonnées,

Figure 21

Aperçu des tiroirs à négatifs (cliché Soline Morinière / DRAC Alsace)

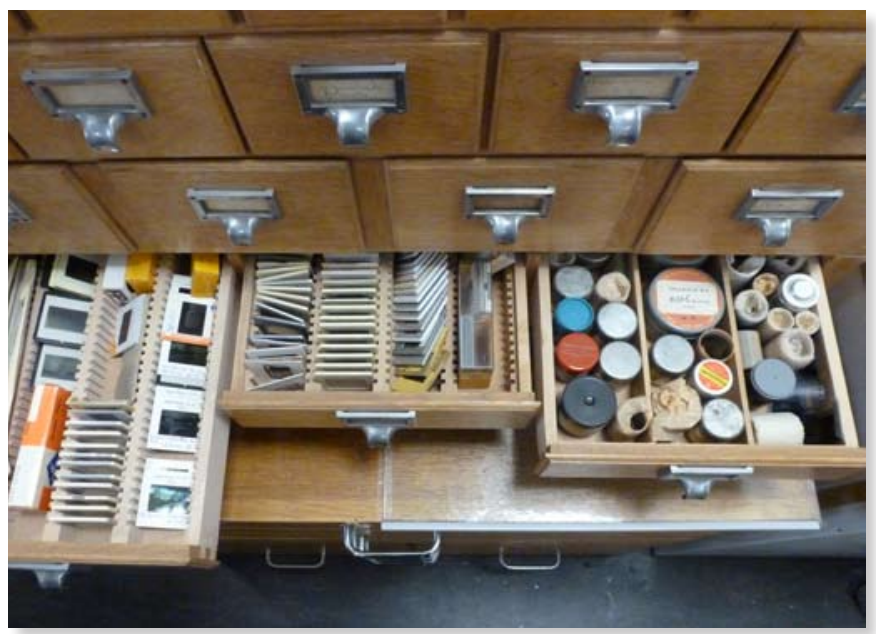




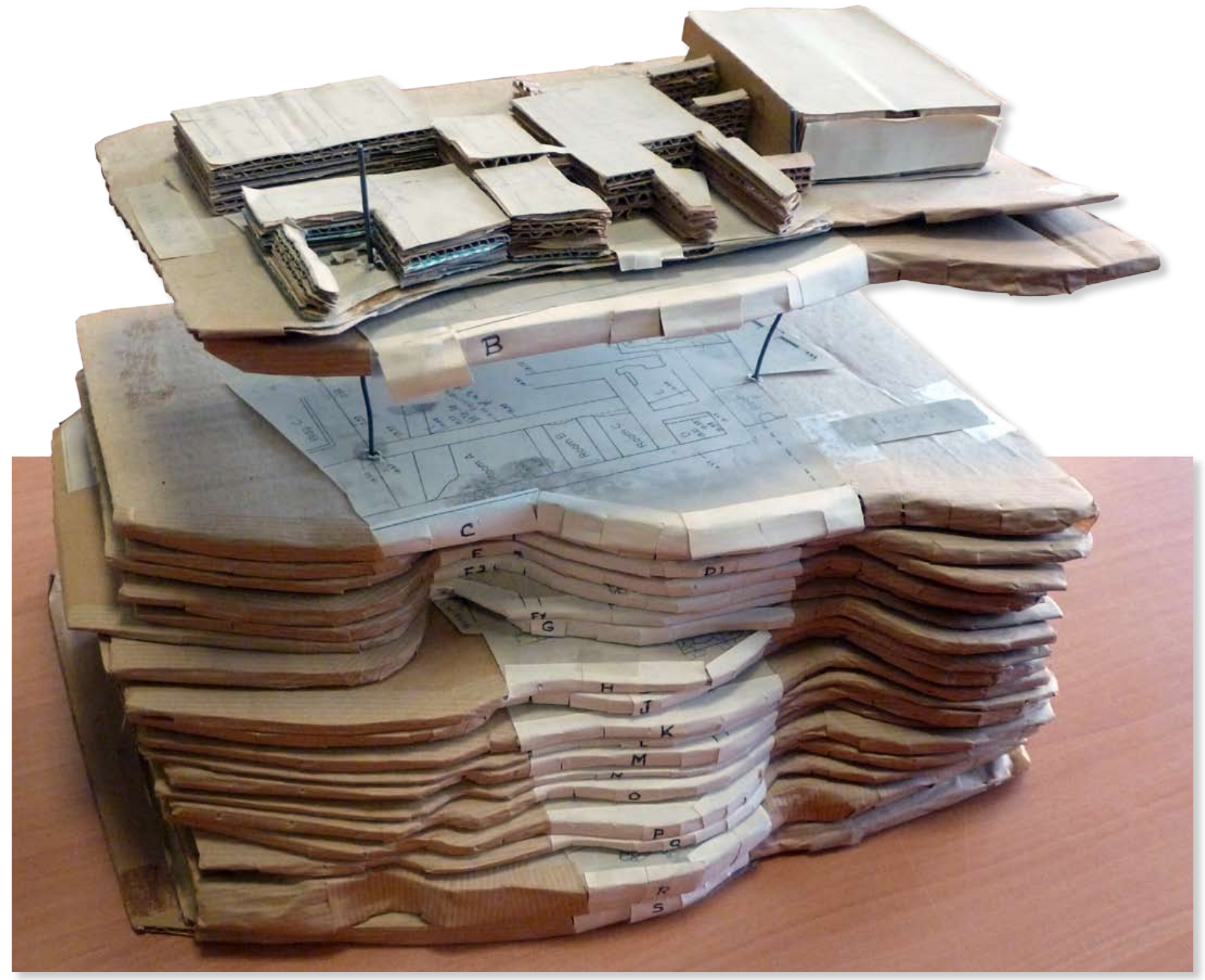

Figure 22

Maquette stratigraphique retrouvée dans les archives Jacqueline Pirenne (cliché : Soline Morinière / DRAC Alsace)

consistent également en de multiples relevés et prises de vues, eux-mêmes conditionnés dans des contenants propres aux années 1950 à 1980 :

- tiroir à négatifs, allant des rouleaux dans des boîtes cylindriques en aluminium, en plastique, à des classeurs spécifiques ;

- tiroirs à encoches pour diapositives ;

- tiroirs à relevés, nombreux et fins, etc.

L'ensemble forme un objet unique, représentatif d'une méthode de travail et de la personnalité d'un chercheur, dont l'intérêt dépasse le cadre strict des données scientifiques qu'il renferme.

\subsubsection{Les plaques de verre}

Les plaques de verre de I'Institut d'Orient ancien, encore classées dans leur tiroir en bois, méritent égale- ment une attention particulière, en raison de l'évolution des techniques photographiques et de I'histoire de la pédagogie.

\subsubsection{Une pépite dans les archives}

Une archive singulière a été découverte dans le fonds Jacqueline Pirenne. Il s'agit de la reconstitution stratigraphique d'un site (à identifier), composée d'une succession de cartons découpés, figurant chacun un niveau d'occupation. Ces plaques sont nommées par la lettre de la couche qu'elles représentent, et accueillent sur la partie supérieure une photocopie du relevé de chaque niveau. Elles s'emboîtent sur deux tiges en métal qui assurent la cohérence de la structure, et permettent de retirer les niveaux au fur et à mesure de la lecture de la stratigraphie du site. 


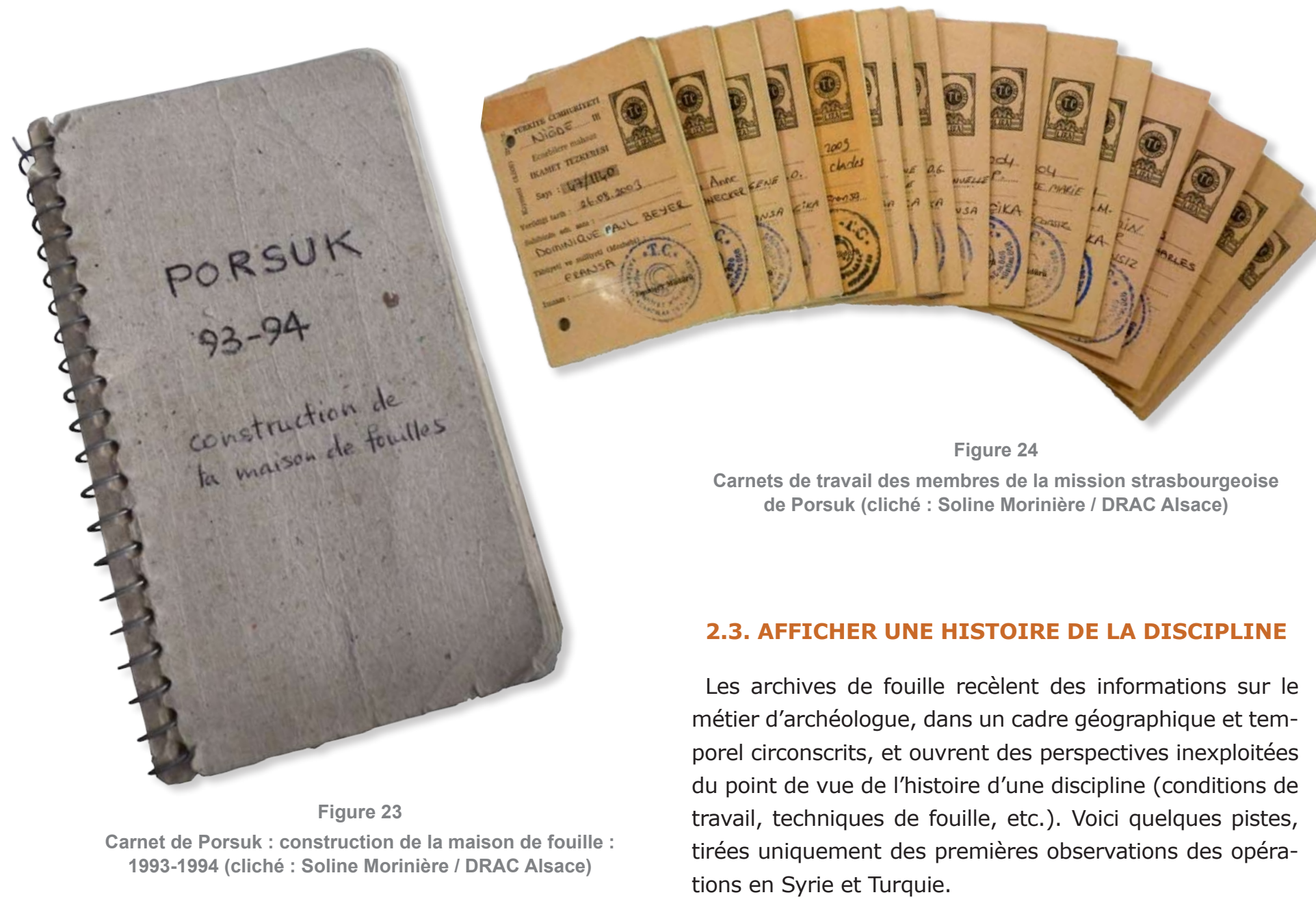

Figure 25

Fiche objet : fusaïole mise au jour en 1996 dans le chantier "Pente ouest" à Tell Mashnaqa (cliché : Soline Morinière / DRAC Alsace)

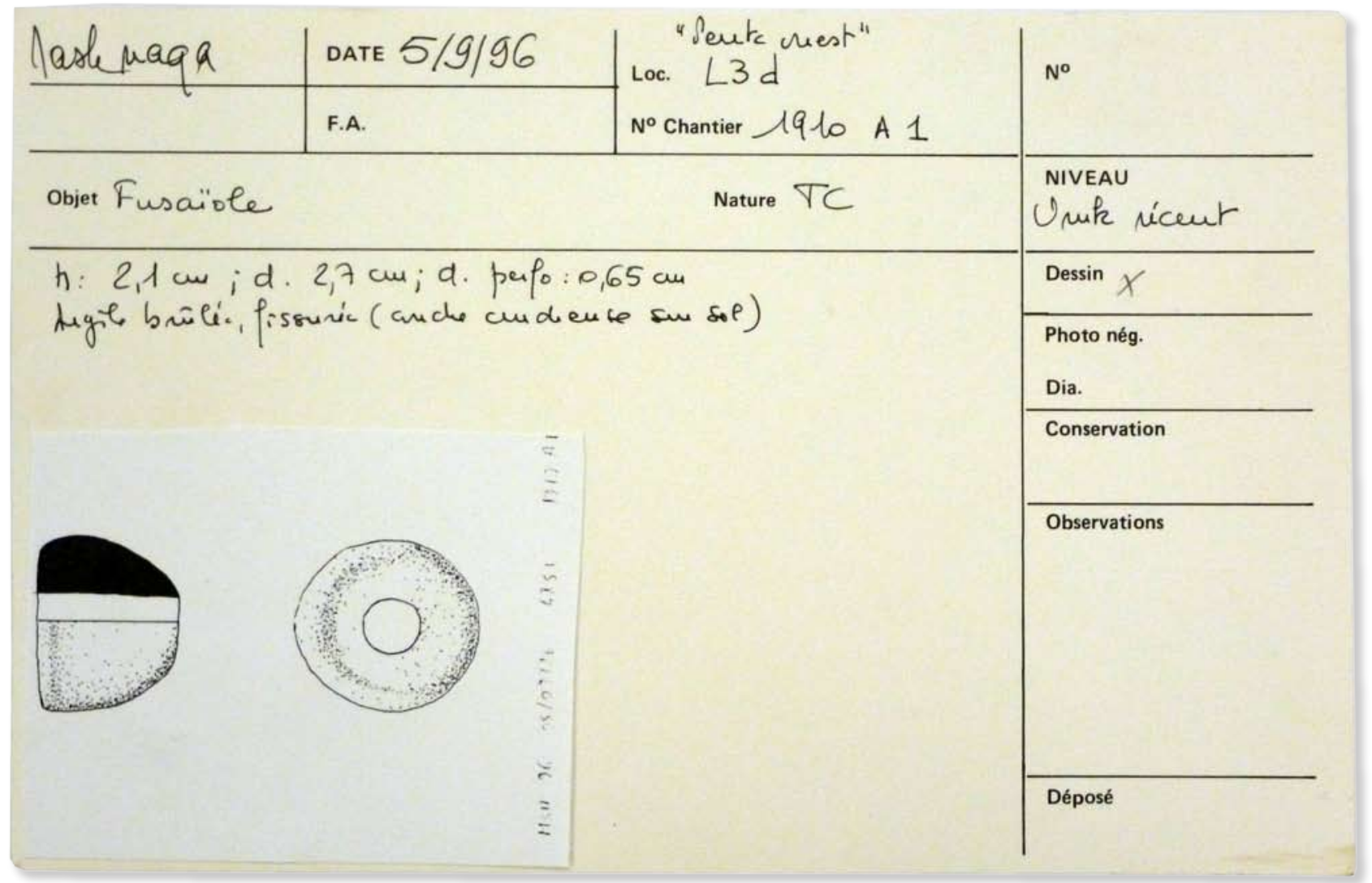




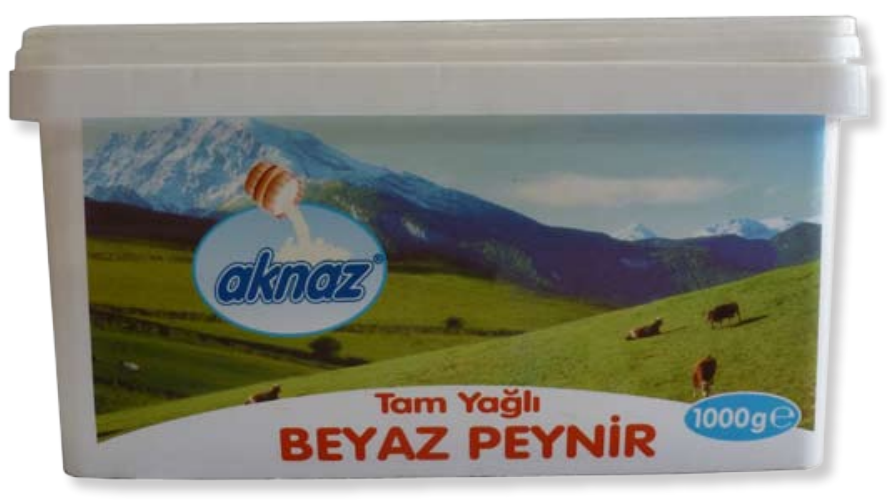

Figure 26

Pot de yaourt recyclé en boîte de prélèvement, fouilles de Porsuk, 2012 (cliché : Soline Morinière / DRAC Alsace)

Ainsi, lors de campagnes pluri-annuelles à l'étranger, il arrive que l'implantation de la mission s'inscrive dans la durée. Aussi, dans les archives de Porsuk avons-nous découvert un carnet relatif à l'édification de la maison de fouille dans les années 1990.

Depuis plusieurs années, les autorités turques demandent aux archéologues d'obtenir un carnet de travail afin de pouvoir fouiller dans le pays. Les archives de Porsuk conservent un lot de carnets de l'équipe.

De nombreux modèles de fiches, de multiples cahiers de fouille sont conservés. Les méthodes d'enregistrement : fiches photo et objet, carnets photo, etc. retracent l'évolution des techniques sur plus d'un demi-siècle.

Signalons ici un point de vue original relatif aux contenants : leur appréhension en tant que témoin des méthodes de travail sur le terrain, notamment à l'étranger, et l'intérêt qu'il peut y avoir à conserver, ou du moins à documenter leur utilisation. Pour exemple, ce prélèvement issu des collections de Porsuk, qui témoigne de la réutilisation des objets du quotidien et de I'adaptabilité des archéologues.

Enfin, plutôt gardées dans les archives personnelles de fouilleurs, les photos d'équipes sur le terrain renseignent sur les conditions de vie lors d'un chantier à l'étranger.

[24] Notamment à la MSH de Dijon : https:// pandor.u-bourgogne.fr/, la MSH René Ginouvès de Nanterre : http://archives.mae.u-paris10.fr/ la MSHS de Toulouse : http://w3.arshs-mshst. univ-tlse2.fr/. Cf. STAHL 2015.

\subsection{LES ARCHIVES DE CHERCHEURS : UNE VALEUR AJOUTÉE POUR L'IMAGE DES INSTITUTIONS}

Des expériences concluantes en matière de gestion archivistique ont déjà été menées dans plusieurs universités [24] et convergent toutes actuellement vers un système de gestion similaire : une description archivistique répondant aux normes internationales en vigueur, ISAD (G) et encodage en XML EAD, pour une diffusion en ligne via une plateforme dédiée associant inventaire et documents numériques ou numérisés. Cette méthode, employée au SRA Alsace depuis 2006, offre au consultant l'accès à plus de 400 instruments de recherche, dont la chaîne de production est réalisée au moyen d'outils libres.

Au vu de ce panorama et des expériences déjà existantes, une coopération entre les membres de I'UMR 7044 Archimède (Université de Strasbourg, CNRS et Ministère de la culture) autour d'un projet d'inventaire et de diffusion des archives de l'archéologie serait largement profitable aux trois entités et, plus largement, à l'accès aux sources primaires de la recherche en général.

\section{Figure 27}

Les bases Pleade en XML EAD de la MAE Rene Ginouvès (en haut) et du SRA Alsace (en bas) : un même système de gestion des archives scientifiques

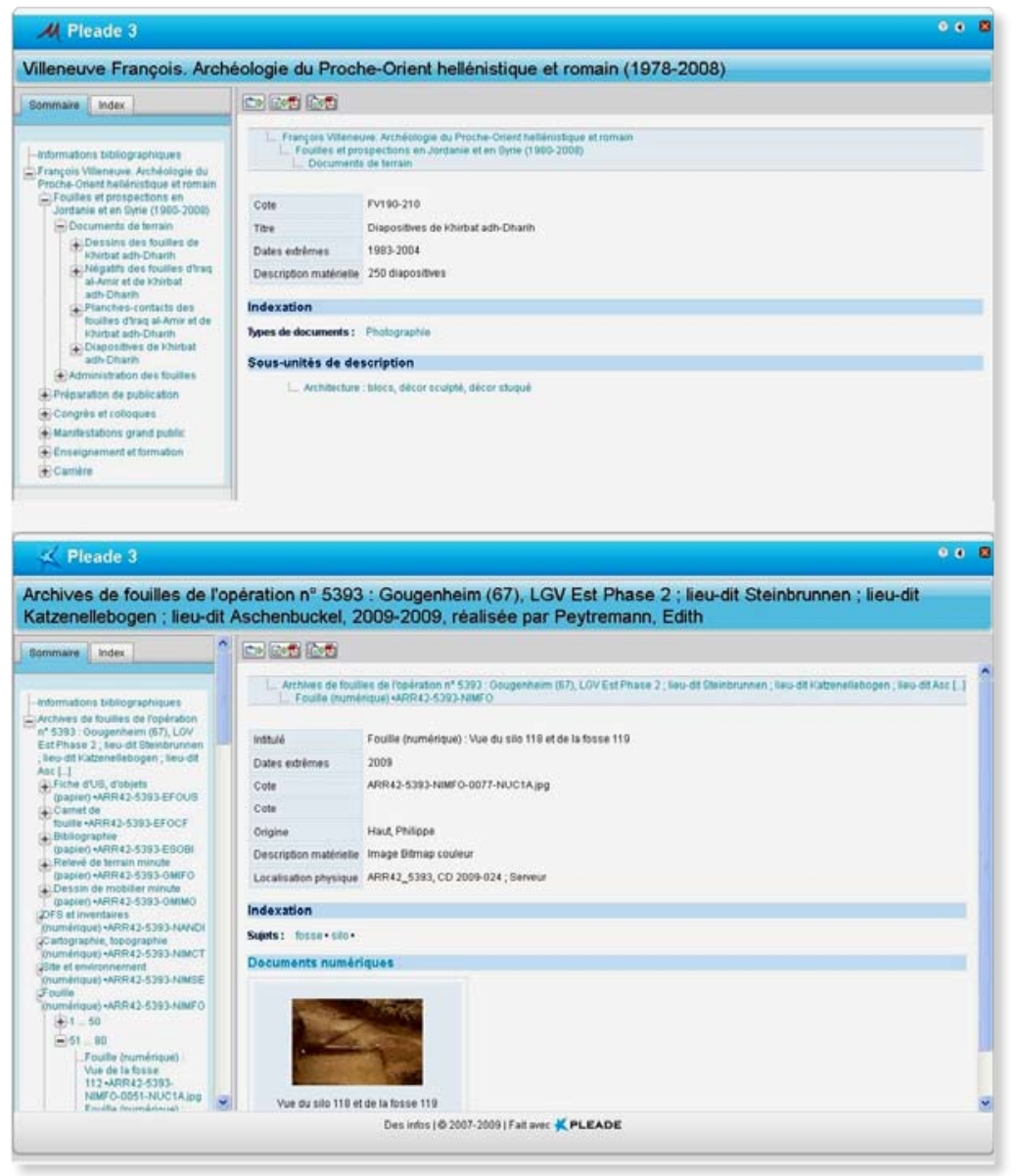




\section{BIBLIOGRAPHIE}

LoRentz, Claude, 2000, Les fonds anciens de I'Université Marc Bloch de Strasbourg : historique, essai d'évaluation et situation générale, mémoire d'étude [en ligne]. URL :

www.enssib.fr/bibliotheque-numerique/documents/1340-les-fonds-anciens-de-l-universite-marc-bloch-de-strasbourg-historique-essai-d-evaluation-et-situation-generale.pdf

Michaelis, Adolf, 1987, Führer durch das archäologische Museum der Kaiser-Wilhelms-Universität Strassburg, Strassburg.

Seyrig, Henri, 1961, «André Maricq (1925-1960) ». Syria 38, p. 350-354 [en ligne]. URL : www.persee.fr/web/revues/home/prescript/article/syria_0039-7946_1961_num_38_3_5556

Staht, Marie, 2015, « Archivage de la documentation de fouille : état des lieux et recommandations à l'usage des archéologues », dans Pascal Butterlin, Julie Patrier, Philippe Quenet (éd.), Mélanges en I'honneur de Dominique Beyer (Subartu), Turnhout, à paraître.

WILL, Ernest, 1991, « Jacqueline Pirenne (1918-1990) ». Syria 68, p. 465-466 [en ligne]. URL :

www.persee.fr/web/revues/home/prescript/article/syria_0039-7946_1991_num_68_1_7231

Wolikow, Serge, 2009, « L'enquête sur les archives de la recherche en sciences humaines et sociales (ARSHS) : premier bilan », Histoire@politique 9, p. 100 [en ligne]. URL : www.cairn.info/revue-histoire-politique-2009-3page-100.htm

\section{ORIENTATION BIBLIOGRAPHIQUE DES SITES}

\section{PORSUK}

Beyer, Dominique, 2007, « Zeyve höyük (Porsuk) : rapport sommaire sur la campagne de fouilles de 2007 », Anatolia antiqua 16, p. 313-344.

Beyer, Dominique, 2008, « Zeyve Höyük (Porsuk), 2006 », Kazı sonuçları toplantısı 29, p. 107-116.

Beyer, Dominique, 2009a, «Zeyve Höyük (Porsuk), rapport sur la campagne de 2008 », Anatolia antiqua 17, p. 317-349.

Beyer, Dominique, 2009b, « Zeyve Höyük (Porsuk), 2007 », Kazı sonuçları toplantısı 30, p. 385-392.

Beyer, Dominique, 2010, «Campagne 2009 de la mission archéologique de Zeyve Höyük (Porsuk) », Anatolia antiqua 18, p. 215-242.

Beyer, Dominique \& Chalier, Isabelle, 2007, « Zeyve Höyük (Porsuk) : rapport sommaire sur la campagne de fouilles de $2006 \gg$, Anatolia antiqua 15, p. 289-314.

Beyer, Dominique, Laroche-Traunecker, Françoise, Patrier, Julie \& Tibet, Aksel, 2012, « Zeyve Höyük - Porsuk : rapport préliminaire sur la campagne $2012 \gg$, Anatolia antiqua 20, p. 177-203.

Beyer, Dominique, Chalier, Isabelle, Kirner, Françoise, Laroche-Traunecker, Françoise \& Tibet, Aksel, 2013, « Zeyve Höyük - Porsuk : rapport préliminaire sur la campagne 2013 », Anatolia antiqua 21, p. 201-234. 


\section{MESKÉNÉ / EMAR}

Beyer, Dominique, 2001, Mission archéologique de Meskéné-Emar : recherches au pays d'Aštata. IV, Les sceaux (Orbis biblicus et orientalis 20), Fribourg.

Weygand, Isabelle, 2012, «Les objets en métal d’Emar (aujourd'hui Meskéné), une ville de Syrie du nord sous domination hittite au Bronze récent : quels courants d'influence ? », Anatolica 38, p. 237-261.

\section{TELL MASHNAQA}

Beyer, Dominique, 1993a, «Mashnaqa 1993: rapport sommaire sur les travaux de la mission archéologique française », Orient-Express 1993/1, p. 13-15.

Beyer, Dominique, 1993b, « Mashnaqa (Syrie) : travaux de la mission archéologique française en août-septembre 1992 », Orient-Express 1993/2, p. 7-8.

Beyer, Dominique, 1995, «Mashnaqa (Syrie) 1994 : rapport sommaire sur les travaux de la mission archéologique française », Orient-Express 1995/2, p. 43-46.

Beyer, Dominique, 1996a, «Mashnaqa», dans Exposition syro-européenne d'archéologie: miroir d'un partenariat : Musée national de Damas, 30 mai-11 juillet 1996 = Syrian European Archaeology Exhibition : working together: Damascus National Museum, May 30th-July 11th 1996, Damas, p. 55-57.

Beyer, Dominique, 1996b, «Mashnaqa 1995 (Syrie) : rapport sur les travaux de la mission française », Orient-Express 1996/1, p. 9-12.

Beyer, Dominique, 1997, «Tell Mashnaqa (Syrie) : travaux de la mission archéologique française 1996 (mission de I'IFAPO Damas) 》, Orient-Express 1997/1, p. 3-5.

Beyer, Dominique, 1998, «Évolution de l'espace bâti sur un site de la vallée du Khabur au IVe millénaire: les fouilles françaises de Mashnaqa », dans Olivier Aurenche \& Michel Fortin (éd.), Espace naturel, espace habité en Syrie du Nord, 10e-2e millénaires av. J-C. (Travaux de la Maison de I'Orient méditerranéen 28), Paris, p. 139-148.

\section{GÜLNAR}

Davesne, Alain \& Laroche-Traunecker, Françoise, 1998, Gülnar I : le site de Meydancikkale, Paris.

\section{LYCIE}

Dardaine, Sylvie \& Frézouls, Edmond, 1985, «Sidyma : étude topographique », Ktèma 10, p. 211-217.

Dardaine, Sylvie \& Longepierre, Daniel, 1985, «Essai de typologie des monuments funéraires de Sidyma (époques lycienne et romaine) 》, Ktèma 10, p. 220-232.

Frezouls, Edmond \& Morant, Marie-José, 1985, «Inscriptions de Sidyma et de Kadyanda (I) 》, Ktèma, 10, p. $233-243$.

Frezouls, Edmond \& Morant, Marie-José, 1986, « Inscriptions de Sidyma et de Kadyanda (II) », Ktèma 11, p. $239-253$.

Frezouls, Edmond, Morant, Marie-José \& Longepierre, Daniel, 1986, « Urbanisme et principaux monuments de Kadyanda », Ktèma 11, p. 225-238.

Jacquemin, Anne \& Morant, Marie-José, 1999, « Inscriptions de Kadyanda », Ktèma 24, p. 289-288.

\section{TELL HARIRI / MARI : TEMPLES, TERRES CUITES ET SCEAUX}

BeYer, Dominique, 2007, « Les sceaux de Mari au IIIe millénaire : observations sur la documentation ancienne et les données nouvelles des villes I et II $\gg$, Akh Purattim 1, p. 231-260.

BeYer, Dominique, 2014, « Les temples de Mari, bilan de 20 ans de travaux au chantier G, (1990-2010) », Syria supplément 2, p. 517-539.

Beyer, Dominique \& Jean-Marie, Marylou, 2007, « Le temple du DA III de la déesse Ninhursag à Mari : les dépôts votifs du Lieu Très Saint », Akh Purattim 2, p. 75-122.

Beyer, Dominique \& Lecompte, Camille, 2014, « La glyptique du secteur du temple d'Ishtar à Mari », dans Sophie Cluzan et Pascal Butterlin (éd.), Voués à Ishtar, Beyrouth, p. 275-282.

Weygand, Isabelle, 1993, « Un piège à Mari », MARI 7, p. 329-337.

Weygand, Isabelle, 2006a, « Les déesses-bustes à Mari et Terqa », dans Pascal Butterin et. al. (éd.), Les espaces syro-mésopotamiens : volume d'hommages offert à Jean Margueron (Subartu 17), Turnhout, p. 439-448.

Weygand, Isabelle, 2006b, «Les terres-cuites de Mari, directions de recherches », Akh Purattim 1, p. $269-278$.

Weygand, Isabelle \& Pic, Marielle, 1983, « Une terre cuite provenant de Mari figurant la déesse Ishtar », MARI 2, p. $201-209$. 
WEYgand, Isabelle, à paraître, «Le thème des buveurs au chalumeau sur les reliefs estampés en terre cuite découverts à Terqa et à Mari », dans Pascal Butterlin, Julie Patrier, Philippe Quenet (éd.), Mélanges en I'honneur de Dominique Beyer (Subartu), Turnhout.

\section{SYRIE DU NORD}

Charpentier, Gérard, Duvette, Catherine, Marillat, Cédric, Piaton, Claudine, 2007, « Présentation des travaux réalisés en Syrie du Nord dans le cadre du programme européen 14 », Résultats du programme de formation à la sauvegarde du patrimoine culturel de Syrie, (Documents d'archéologie syrienne 9), Damas.

Duvette, Catherine, 1999, «Technique de relevé et exploitation des données adaptées aux vestiges proto-byzantins de la Syrie du Nord », dans Actes de la table ronde sur les techniques de relevé d'élévation, Lyon, p. 157-161.

Duvette, Catherine, 2012, « Le ğebel Zawiyé (Massif calcaire de la Syrie du Nord) : I'architecture domestique, reflet d'une évolution démographique », dans Mondes ruraux en Orient et en Occident 1, Antiquité tardive 20, p. 94-100.

Duvette, Catherine, Piaton, Claudine, à paraître, «Évolution d'une technique de construction et croissance des villages du ğebel Zawiyé », Topoi supplément 12.

Duvette, Catherine, Charpentier, Gérard, Piaton, Claudine, à paraître, «Maisons paysannes d'un village d'Apamène, Serğilla (IVe-VIe siècles - Massif calcaire de la Syrie du Nord)», dans Mondes ruraux en Orient et en Occident 2, Antiquité tardive 21.

Tate, Georges, Abdulkarim, Maamoun, Charpentier, Gérard, Duvette, Catherine, Piaton, Claudine, 2013, Serğilla, village d'Apamène, Beyrouth-Damas. 